\title{
Impact of Chlorine on the Internal Transition Rates and Excited States of the Thermally Delayed Activated Fluorescence Molecule 3CzClIPN
}

\author{
Martin Streiter, ${ }^{1}$ Tillmann G. Fischer, ${ }^{2}$ Christian Wiebeler, ${ }^{3,4}$ Sebastian \\ Reichert, ${ }^{1}$ Jörn Langenickel, ${ }^{5}$ Kirsten Zeitler, ${ }^{2}$ and Carsten Deibel ${ }^{* 1}$ \\ ${ }^{1}$ Institut für Physik, Technische Universität Chemnitz, 09126 Chemnitz, Germany \\ ${ }^{2}$ Institut für Organische Chemie, Universität Leipzig, 04103 Leipzig, Germany \\ ${ }^{3}$ Institut für Analytische Chemie, Universität Leipzig, 04103 Leipzig, Germany \\ ${ }^{4}$ Leibniz-Institut für Oberflächenmodifizierung (IOM), 04318 Leipzig, Germany \\ ${ }^{5}$ Zentrum für Mikrotechnologien, Technische Universität Chemnitz, 09126 Chemnitz, Germany
}

NOTE: This document is the Accepted Manuscript version of a Published Work that appeared in final form in The Journal of Physical Chemistry C, copyright American Chemical Society after peer review and technical editing by the publisher. To access the final edited and published work see DOI: https://doi.org/10.1021/acs.jpcc.0c03341

ABSTRACT: We analyze internal transition rates and the singlet-triplet energy gap of the thermally activated delayed fluorescence (TADF) molecule $3 \mathrm{CzClIPN}$, which recently was introduced as an efficient photocatalyst. Distribution and origin of the non-monoexponential decays, which are commonly observed in TADF films, are revealed by analysis of transient fluorescence with an inverse Laplace transform. A numerically robust global rate fit routine, which extracts all relevant TADF parameters by modeling the complete set of data, is introduced. To compare and verify the results, all methods are also applied to the well-known 4 CzIPN. The influence of the molecular matrix is discussed by embedding low concentrations of TADF molecules in polystyrene films. Finally, quantum chemical calculations are compared to the experimental results to demonstrate that the chlorine atom increases the charge transfer character of the relevant states, resulting in a reduction of the singlet-triplet energy gap.

Keywords: TADF, 4CzIPN, 3CzCIIPN, reverse intersystem crossing, transition orbitals

\section{INTRODUCTION}

State-of-the-art materials utilized in organic lightemitting diodes (OLED) and photocatalysis (PC) usually contain metal-complexes with rare elements such as iridium and ruthenium. ${ }^{1-4}$ Production cost, element scarcity and questions of environmentally friendly mining led to efforts in synthesizing metal-free molecules with comparable and therefore competitive photophysical and chemical properties. In OLED technology, internal quantum efficiencies of $100 \%$ can be realized with traditional metal-complexes by triplet recombination (phosphorescence), overcoming the limitation of maximal $25 \%$ internal quantum efficiency set by singlet spin statistics. ${ }^{5}$ By converting triplet states into singlet states, so-called TADF (thermally activated delayed fluorescence) materials can - without the necessity of a metal center - equally achieve up to $100 \%$ internal quantum efficiency. ${ }^{6}$ In addition to prompt fluorescence emission from the singlet $S_{1}$ state back into the $S_{0}$ ground state (referred to as $\mathrm{PF}$ in the following), the $T_{1}$ triplet state (populated by intersystem crossing with rate $k_{\text {ISC }}$ ) can repopulate the $S_{1}$ state by reverse intersystem crossing $\left(k_{\text {RISC }}\right)$, leading to delayed fluorescence (DF). TADF materials are characterized by a donor-acceptor structure which also can be realized by metal-free molecules. Localized wavefunctions on donor and acceptor sites are spatially and energetically well separated, usually by large dihedral angles between donor and acceptor groups. This design leads to a decrease of the energy difference $\Delta E_{\mathrm{ST}}$ between excited
$S_{1}$ and $T_{1}$ state. These three parameters are theoretically connected by detailed balance in equation (1). ${ }^{7}$ The equation suggests that small $\Delta E_{\mathrm{ST}}$ are favored in OLED design because of efficient singlet-from-triplet conversion as well as important in PC as an indicator of a strong charge transfer state character of the excited state. However, when $\Delta E_{\mathrm{ST}}$ is too low, the exchange integral between ground and excited state approaches zero, leading to low absorption. ${ }^{8}$ Therefore, in both, OLED and PC applications, also parameters such as absorption, nonradiative losses, quantum yield and $k_{\text {RISC }}$ need to be considered.

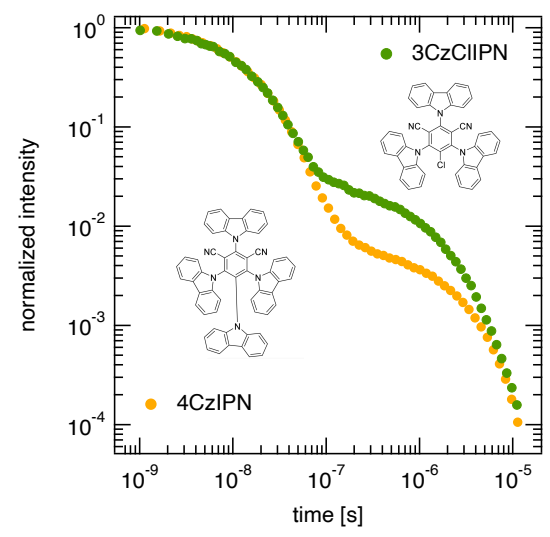

FIG. 1. Prompt and delayed fluorescence decay of $4 \mathrm{CzIPN}$ and $3 \mathrm{CzClIPN}$ films at room temperature. 


$$
k_{\mathrm{RISC}}=k_{\mathrm{ISC}} \exp \left(-\frac{\Delta E_{\mathrm{ST}}}{k_{\mathrm{B}} T}\right) .
$$

One of the most studied TADF molecule is $4 \mathrm{CzIPN}$ (1,2,3,5-tetrakis(carbazol-9-yl)-4,6-dicyanobenzene), which was introduced by Adachi et al. ${ }^{6}$ The four carbazole groups act as an electron donor attached to the electron accepting 4,6-dicyanobenzene core at an angle of $60^{\circ}$. Adachi et al. first demonstrated its efficient properties as a green OLED emitter. ${ }^{6}$ As a photocatalyst, $4 \mathrm{CzIPN}$ shows strong oxidative and reductive ground state potentials. Recently it was shown that modification of $4 \mathrm{CzIPN}$ to $3 \mathrm{CzClIPN}$ increases the oxidative ground state potential demonstrating $3 \mathrm{CzClIPN}$ as a potential alternative to the well-established, oxidizing photocatalyst $\operatorname{Ir}\left(\mathrm{dF}-\mathrm{CF}_{3} \text {-ppy }\right)_{2}(\mathrm{dtbbpy})\left(\mathrm{PF}_{6}\right){ }^{9,10}$ While the influence of chlorine on TADF molecules is only partly discussed in the literature ${ }^{11,12}$, little is known about the photophysical properties of the photocatalytically potent molecule $3 \mathrm{CzClIPN}$. In this paper, we elucidate how chlorine influences the internal transition rates and excited state energies by examining temperature-dependent fluorescence transients with two improved algorithms. Quantum chemical calculations and wavefunction analysis support the experimental results and explain the difference between the two molecules in detail.

\section{METHODS}

Photons emitted via $\mathrm{PF}$ and $\mathrm{DF}$ are energetically indistinguishable, as they both originate from $S_{1}$ (see SI). However, in time-dependent measurements, PF and DF can be separately analyzed as they occur on different timescales with different intensities. Precise and robust routines for measuring, evaluating and simulating TADF parameters are in the focus of current research. ${ }^{8,13-17}$ Figure 1 shows the time-dependent fluorescence and chemical structure of the two molecules in focus of this study, $4 \mathrm{CzIPN}$ and $3 \mathrm{CzClIPN}$.

We compare two improved evaluation methods based on the work of Dias ${ }^{14}$, Berberan-Santos ${ }^{18,19}$, Penfold ${ }^{20}$, $\mathrm{Scholz}^{21}$, Haase ${ }^{22}$ and respective co-authors. Both methods require only fluorescence transients without additional measurements of quantum yields, triplet quenching or phosphorescence. They are therefore experimentally straightforward and sensitive, allowing analysis of highly diluted films with weak emission towards projected single molecule measurements.

Dias et al. showed, that $k_{\text {RISC }}$ can be estimated from fluorescence transient measurements when the yields of intersystem crossing and reverse intersystem crossing of the material are high. ${ }^{14}$ More specifically, for a DF/PF ratio larger than $\approx 4$ (which can be determined from the integrals of both regimes in the transient fluorescence signal) and an assumed reverse intersystem crossing yield approaching $100 \%, k_{\mathrm{RISC}}$ can be estimated from transient measurements with a DF lifetime $\tau_{\mathrm{DF}}$ by:

$$
k_{\mathrm{RISC}}=\frac{1}{\tau_{\mathrm{DF}}}\left(1+\frac{\int_{0}^{\infty} D F \mathrm{~d} t}{\int_{0}^{\infty} P F \mathrm{~d} t}\right) .
$$

To determine $\tau_{\mathrm{DF}}$ and the integral $\mathrm{DF} / \mathrm{PF}$ ratio $(\approx 5$ for $4 \mathrm{CzIPN}$ and $\approx 6$ for $3 \mathrm{CzClIPN}$, therefore equation $(2)$ was applicable), we analyzed our data with the RegSLapS algorithm which utilizes an inverse Laplace transform. ${ }^{23}$ The spectral function $g$ including the effective rates $k_{i}$ for each component in the multiexponential decay $L$ can be obtained by calculating the inverse Laplace transform of:

$$
L(t, T)=\int_{0}^{\infty} g(k(T)) \exp (-k(T) t) \mathrm{d} k .
$$

The solution $g$ contains information about the amplitudes $A_{i}$, effective rates $k_{i}$ as well as the necessary number of components $i$. Subsequently, every decay can be fitted with the sum of multiple exponential functions (see $\mathrm{SI}$,

$$
L(t, T)=\sum_{i} A_{i} \exp \left(-k_{i} t\right)
$$

This analysis is illustrated in figure 2 for the transient fluorescence of a neat $4 \mathrm{CzIPN}$ film and also applied to $3 \mathrm{CzClIPN}$ at room temperature. Since solving of equation (3) is an ill-posed problem, the experimental data has to be almost noise free to avoid instability of the solution $g$. Additionally, uniqueness could not be given as different spectral functions $g$ can have nearly the same Laplace transform. Therefore, we used an extended version of the Tikhonov's regularization as described by Reichert at al..$^{23}$ to search for the most stable and reasonable solutions.

With the integral of $A \exp (-k t)$ being $A k^{-1}$ and an effective DF rate $^{24}$ (see SI), $k_{\text {RISC }}$ was calculated from the inverse Laplace transform with

$$
k_{\mathrm{RISC}}=\left\langle k_{\mathrm{DF}}\right\rangle\left(1+\frac{\sum A_{i} k_{i \mathrm{DF}}^{-1}}{\sum A_{j} k_{j \mathrm{PF}}^{-1}}\right) .
$$

Temperature-dependent values of $k_{\mathrm{RISC}}$ were evaluated according to equation (5), and the $k_{\mathrm{RISC}}(T)$ plots (see SI) were then fitted with

$$
k_{\mathrm{RISC}}=k_{\mathrm{A}} \exp \left(-\frac{\Delta E_{\mathrm{ST}}}{k_{\mathrm{B}} T}\right) .
$$

Note, that the prefactor $k_{\mathrm{A}}$ is described differently in the literature, as discussed later. ${ }^{6,7,16,25}$ We refer to this evaluation method as Laplace fit throughout the paper. 


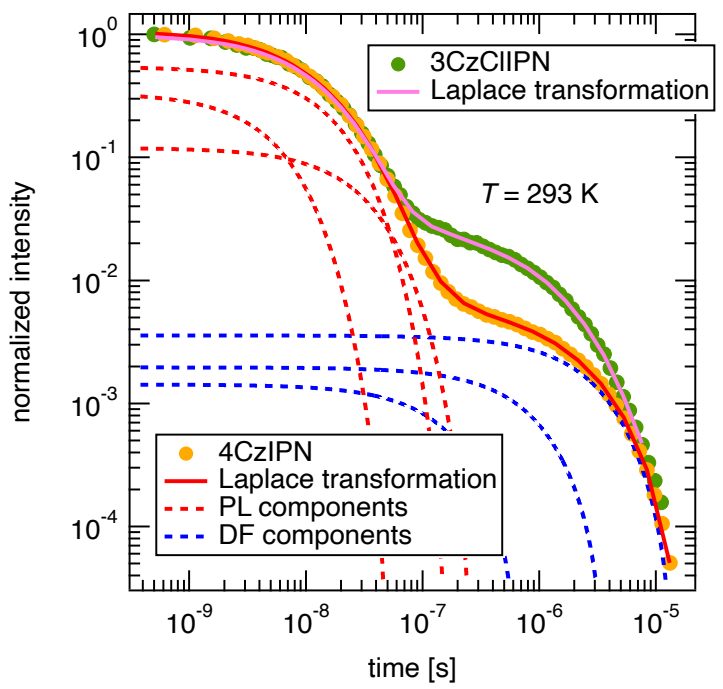

FIG. 2. Examplary single components of the inverse Laplace transform algorithm. Resulting fits for neat $4 \mathrm{CzIPN}$ and $3 \mathrm{CzClIPN}$ films at room temperature.

Next, we discuss our second method, the global rate fit, which minimizes a global differential rate equation system to the complete, temperature-dependent data set. Haase et al. showed that extracting all relevant internal transition rates is possible by fitting the commonly assumed coupled differential equation system $(7-8)$ to the data, ${ }^{22}$

$$
\begin{aligned}
& \dot{S}_{1}(t)=-\left(k_{\mathrm{F}}+k_{\mathrm{ISC}}\right) S_{1}(t)+k_{\mathrm{RISC}} T_{1}(t) \\
& \dot{T}_{1}(t)=k_{\mathrm{ISC}} S_{1}(t)-k_{\mathrm{RISC}} T_{1}(t) .
\end{aligned}
$$

Here, $k_{\mathrm{F}}$ is the sum of radiative and nonradiative depopulation rates of the $S_{1}$ singlet state. The prerequisite for this model is the reduction of the TADF mechanism to a three-level system involving only $S_{0}, S_{1}, T_{1} \cdot{ }^{20}$ This ignores the influence of disorder because in an organic molecule film, a broad distribution of states is present. Additionally, recombination from $T_{1}$ (nonradiative or as phosphorescence) is neglected, as well as a possible contribution from higher excited triplet states. ${ }^{26}$ The influence of each of these simplifications will be explained in detail later.

The solution of equation system (7-8) equals the sum of two monoexponential functions with different amplitudes and rates, which is insufficient for modeling our data, which deviates from monoexponential decay especially during the PF. From the inverse Laplace transform analysis, we concluded that the non-monoexponential $\mathrm{PF}$ decay can be well described with two distinct rates in most cases. These decay characteristics are caused by dynamic and static inhomogeneities of the molecules' conformation and environment in film as discussed later in more detail. ${ }^{27,28}$ We assumed that $k_{\mathrm{F}}$ and $k_{\mathrm{ISC}}$ can be treated as temperature-independent parameters because normalized PF decay showed no correlation with temperature. The advantage of fitting a rate equation system to the data, as demonstrated by Haase et al., is the direct extraction of all internal rates. However, this evaluation method also consists of two steps: kinetic modeling and fitting of $k_{\mathrm{RISC}}(T)$. We believe that a numerically more robust method is the direct extraction of all relevant parameters from the complete, temperaturedependent data set by global rate fitting in one step. We implemented an algorithm that includes the sum of two singlet states (as predicted by our Laplace evaluation which showed two distinct rate peaks of the fluorescence as shown in the SI). Both singlet states $S_{1,2}$ had a starting population of $N_{1,2}$ after excitation and were depopulated with rates $k_{\mathrm{F}^{1,2}}, k_{\mathrm{ISC}}$ and repopulated with $k_{\mathrm{RISC}}$, depending on the triplet population $T_{1,2}(t)$, where $T_{1,2}(0)=0$.

$$
\begin{aligned}
\dot{S}_{1,2}(t) & =-\left(k_{\mathrm{F}^{1,2}}+k_{\mathrm{ISC}}\right) S_{1,2}(t)+k_{\mathrm{RISC}} T_{1,2}(t) \\
\dot{T}_{1,2}(t) & =k_{\mathrm{ISC}} S_{1,2}(t)-k_{\mathrm{RISC}} T_{1,2}(t) \\
k_{\mathrm{RISC}} & =k_{\mathrm{A}} \exp \left(-\frac{\Delta E_{\mathrm{ST}}}{k_{\mathrm{B}} T}\right)
\end{aligned}
$$

\section{RESULTS AND DISCUSSION}

Figure 3 shows the results of both evaluation methods for neat $4 \mathrm{CzIPN}$ and $3 \mathrm{CzClIPN}$ films, respectively. Fit results are summarized in table 1 . The Laplace fit algorithm fitted all data sets well on the full time scale of the decay while global rate fitting showed small deviations in the DF part of the decay after $1 \mu \mathrm{s}$. We also tested more complex rate equation systems with multiple singlet and triplet states ${ }^{26}$ in order to yield precise fits of the DF part, but found the process to be over-parameterized quickly, allowing very good fits but no robust way of extracting the effective values or possible distribution characteristics. This is shown and discussed in detail in the SI.

We now discuss the results for neat films and compare both evaluation methods with our calculated values and the literature. Both evaluation methods delivered matching values $\Delta E_{\mathrm{ST}}=(54 \pm 5) \mathrm{meV}$ for a neat $4 \mathrm{CzIPN}$ film. Depending on film matrix (or solvent), but also measurement technique and evaluation method, values between $30 \mathrm{meV}$ and $140 \mathrm{meV}$ are reported for $4 \mathrm{CzIPN}$ in the literature. ${ }^{6,26,29-34}$ However, in neat film with a similar experiment, Olivier et al. measured $42 \mathrm{meV}$ (and simulated value: $60 \mathrm{meV}$ ), which is close to our result. ${ }^{30} \mathrm{In}$ $3 \mathrm{CzClIPN}$, we found $\Delta E_{\mathrm{ST}}=(33 \pm 8) \mathrm{meV}$. Note, that this is the first value of $\Delta E_{\mathrm{ST}}$ reported for $3 \mathrm{CzClIPN}$. The error in determining $\Delta E_{\mathrm{ST}}$ increases for low $\Delta E_{\mathrm{ST}}$ values because the temperature dependence of $\mathrm{DF}$ becomes small compared to the dynamic range of the complete decay. The mechanism behind the chlorine-induced decrease of $\Delta E_{\mathrm{ST}}$ is explained later in the molecular 


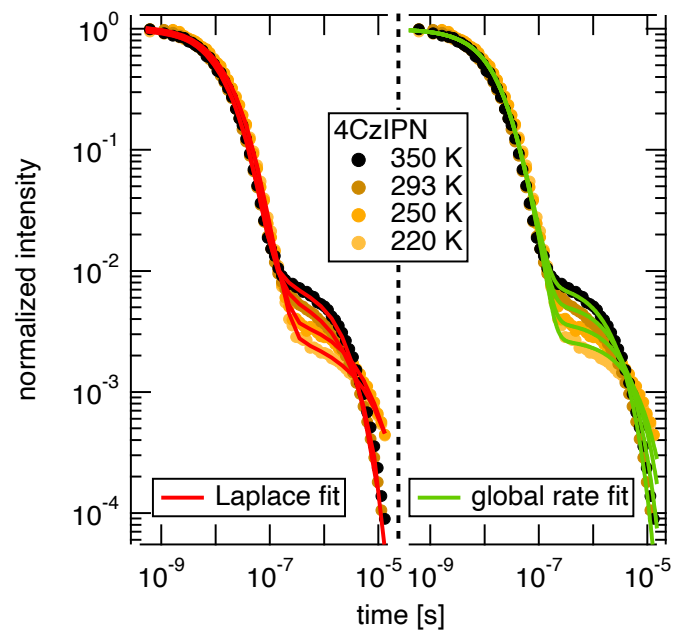

(a) 4 CzIPN, neat film

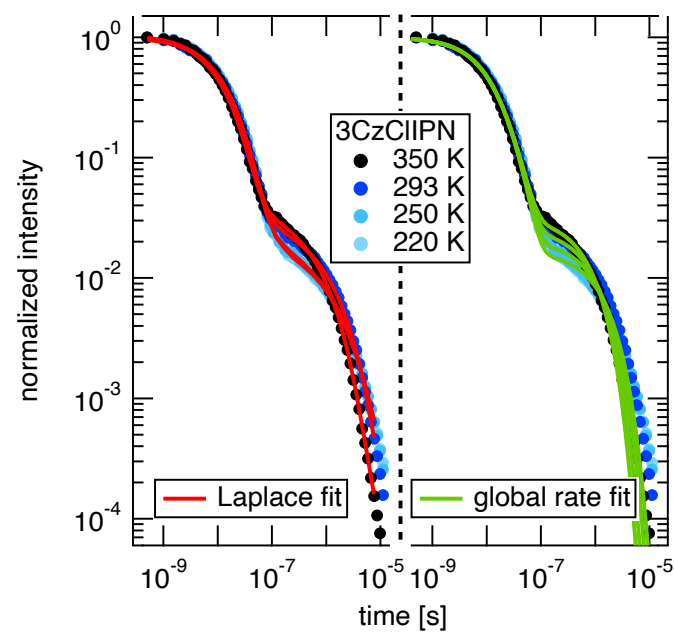

(b) $3 \mathrm{CzClIPN}$, neat film

FIG. 3. Comparison of both fit methods applied to temperature-dependent transient fluorescence decay of neat TADF films

\begin{tabular}{lllccc} 
& film & 4 CzIPN & \multicolumn{3}{c}{$3 \mathrm{CzClIPN}$} \\
method & & neat & in PS & neat & in PS \\
\hline \hline Laplace fit & & & & \\
$\Delta E_{\mathrm{ST}}$ & {$[\mathrm{meV}]$} & 54 & 65 & 34 & 38 \\
$k_{\mathrm{RISC}}$ & {$\left[10^{6} \mathrm{~s}^{-1}\right]$} & 0.6 & 0.7 & 2.1 & 1.8 \\
$k_{\mathrm{A}}$ & {$\left[10^{6} \mathrm{~s}^{-1}\right]$} & 5.1 & 8.7 & 7.6 & 8.9 \\
$\left\langle\tau_{\mathrm{PF}}\right\rangle$ & {$[\mathrm{ns}]$} & 15 & 14 & 16 & 12 \\
\hline Global & rate fit & & & & \\
$\Delta E_{\mathrm{ST}}$ & {$[\mathrm{meV}]$} & 54 & 62 & 33 & 30 \\
$\left\langle k_{\mathrm{F}}\right\rangle$ & {$\left[10^{6} \mathrm{~s}^{-1}\right]$} & 14 & 18 & 17 & 12 \\
$k_{\mathrm{RISC}}$ & {$\left[10^{6} \mathrm{~s}^{-1}\right]$} & 0.7 & 0.8 & 3.9 & 2.6 \\
$k_{\mathrm{A}}$ & {$\left[10^{6} \mathrm{~s}^{-1}\right]$} & 6.2 & 9.4 & 11 & 8.5 \\
$k_{\mathrm{ISC}}$ & {$\left[10^{6} \mathrm{~s}^{-1}\right]$} & 16 & 21 & 31 & 37 \\
\hline \hline PLQY & {$[\%]$} & $\mathrm{n} / \mathrm{a}$ & 99 & $\mathrm{n} / \mathrm{a}$ & 54
\end{tabular}

TABLE I. Experimental results, $k_{\mathrm{RISC}},\left\langle k_{\mathrm{F}}\right\rangle$ and $\left\langle\tau_{\mathrm{PF}}\right\rangle$ at $293 \mathrm{~K}$. PLQY for neat films was not measured due to strong reabsorption.

orbital calculation part. As explained in the introduction, internal transition rates are connected to the $S_{1}$ and $T_{1}$ energies as well as to their relative position. In the literature, $k_{\mathrm{ISC}}$ values between $(1-7) \cdot 10^{7} \mathrm{~s}^{-1}$ are reported. ${ }^{6,26,29-31,35,36}$ At room temperature, $k_{\mathrm{RISC}}$ values between $(6-12) \cdot 10^{5} \mathrm{~s}^{-1}$ are reported..$^{6,30,31,36,37}$ Our results lie between these values (table I). With the global rate fit method, it is possible to simultaneously determine $k_{\mathrm{A}}$ and $k_{\mathrm{ISC}}$. This is enabled by globally fitting for the optimized and temperature-independent $k_{\mathrm{ISC}}$. Within our temperature range between $220 \mathrm{~K}$ and $350 \mathrm{~K}$, we assume $k_{\text {ISC }}$ to be constant. ${ }^{35}$ As shown in table 1 , the prefactor $k_{\mathrm{A}}$ was extracted with very good consistency by both evaluation methods. However, the global rate

fit revealed that the actual $k_{\mathrm{ISC}}$ is higher. Such results can also be found in the literature, when comparing published $k_{\text {ISC }}$ values to the actual prefactor in the according $k_{\text {RISC }}(T)$ plots. The difference between $k_{\text {ISC }}$ and $k_{\mathrm{A}}$ can be interpreted as intramolecular transition pathways which deviate from detailed balance, such as nonradiative $\operatorname{losses}^{30}$, spin-orbit coupling ${ }^{25}$, exciton diffusion ${ }^{29}$ and dynamic asymmetries which result from internal reorganization of the excited charge transfer state. The absolute photoluminescence quantum yield (PLQY) of fluorophores (given by the ratio of emitted/absorbed photons, see SI for details) is dependent on a variety of parameters, such as molecular environment. To avoid reabsorption and fluorophore-fluorophore interaction, we measured the PLQY by embedding $1 \mathrm{wt} \%$ of TADF emitters in a PS matrix, a technique which is known from single molecule spectroscopy. Note, that the spectral characteristics of both, photoluminescence and electroluminescence spectra of $4 \mathrm{CzIPN}$ and $3 \mathrm{CzClIPN}$ are similar, as shown in the SI. We measured a PLQY of $(99 \pm 1) \%$ for $4 \mathrm{CzIPN}$ and $(54 \pm 5) \%$ for $3 \mathrm{CzClIPN}$. Adachi et al. demonstrated that a reduction of the number of carbazole groups and change of substituents can decrease PLQY. ${ }^{6}$ The increased spin-orbit coupling caused by chlorine ${ }^{11}$ may additionally introduce nonradiative transition pathways, thus lowering the PLQY. To examine how nonradiative depopulation of $T_{1}$ would affect the transients, we performed a global rate fit by adding a nonradiative pathway $T_{1} \rightarrow S_{0}$ with rate $\left(k_{\text {nr }}^{T}\right)$ :

$$
\dot{T}_{1}(t)=k_{\mathrm{ISC}} S_{1}(t)-k_{\mathrm{RISC}} T_{1}(t)-k_{\mathrm{nr}}^{T} T_{1}(t) .
$$

This approach showed that high $k_{\mathrm{nr}}^{T}$ rates would lead to a faster DF decay than we experimentally observed. Therefore, $S_{1} \rightarrow S_{0}$ is probably the main nonradiative 
transition channel in $3 \mathrm{CzClIPN}$. Compared to $4 \mathrm{CzIPN}$, we also found a reduced PLQY of $3 \mathrm{CzClIPN}$ in solution (see SI).

To understand the molecular origin of the difference in $\Delta E_{\mathrm{ST}}$ between $4 \mathrm{CzIPN}$ and $3 \mathrm{CzClIPN}$, we performed quantum chemical calculations either based on density functional theory (DFT) or with Post-Hartree-Fock methods. A more detailed discussion of our calculations and in particular the results from regular time-dependent DFT (TD-DFT) calculations with and without TammDancoff approximation (TDA) can be found in the SI. Here, the focus of the presentation will be on the results from simplified TD-DFT (sTD-DFT) and simplified TDA (sTDA), which will be assessed via comparison with results from approximate coupled cluster singles and doubles (CC2) calculations with and without spin-component scaling (SCS), see table II.

\begin{tabular}{lclllll} 
& $4 \mathrm{czIPN}$ & \multicolumn{5}{c}{$3 \mathrm{CzClIPN}$} \\
& $\begin{array}{l}\Delta E_{\mathrm{ST}} \\
\text { method }\end{array}$ & $S_{1}$ & $T_{1}$ & $\Delta E_{\mathrm{ST}}$ & $S_{1}$ & $T_{1}$ \\
\hline B3LYP & {$[\mathrm{eV}]$} & {$[\mathrm{eV}]$} & {$[\mathrm{meV}]$} & {$[\mathrm{eV}]$} & {$[\mathrm{eV}]$} \\
sTD-DFT & 46 & 2.411 & 2.365 & 41 & 2.341 & 2.300 \\
sTDA & 56 & 2.421 & 2.365 & 47 & 2.347 & 2.300 \\
\hline CAM-B3LYP & & & & & & \\
sTD-DFT & 77 & 3.056 & 2.979 & 49 & 3.013 & 2.964 \\
sTDA & 87 & 3.067 & 2.980 & 54 & 3.019 & 2.965 \\
\hline Post-HF & & & & & & \\
CC2 & 43 & 2.842 & 2.798 & 22 & 2.847 & 2.824 \\
SCS-CC2 & 38 & 3.179 & 3.140 & 16 & 3.204 & 3.188
\end{tabular}

TABLE II. Vertical singlet-triplet gaps and energies of the first excited singlet and triplet states relative to the optimized ground state.

The central quantity in our analysis is the energy difference between the $S_{1}$ and $T_{1}$ states for the ground state optimized geometries, i.e. the vertical $\Delta E_{\mathrm{ST}}$. For a more rigorous comparison with experiments, the energy difference of these two states in the corresponding minima should be determined, i.e. the adiabatic $\Delta E_{\mathrm{ST}}{ }^{38}$ Given the similarity of the two states for $4 \mathrm{CzIPN}$ and $3 \mathrm{CzClIPN}$, we expect that the adiabatic singlet-triplet gap can be approximated by its vertical counterpart, which has been proposed by Penfold as well as by Tian et $a l .{ }^{38,39}$ This is further corroborated by two theoretical studies of TADF molecules that reported similar trends for both definitions of $\Delta E_{\mathrm{ST}} \cdot{ }^{40,41}$

The unscaled CC2 calculations yield values for the vertical $\Delta E_{\mathrm{ST}}$ that underestimate the experimental ones by ca. $10 \mathrm{meV}$. Nonetheless, the difference of this energy between the two compounds of $22 \mathrm{meV}$ is close to its experimental counterpart. The application of SCS results in further lowering of both energies by around $5 \mathrm{meV}$, so the difference remains nearly constant. Overall, unscaled CC2 calculations to determine vertical $\Delta E_{\mathrm{ST}}$ yield the best match with experiment among the employed meth- ods (see the SI for a complete overview).

In case of the sTD-DFT/sTDA calculations, the results for individual $\Delta E_{\mathrm{ST}}$ from sTD-DFT based on B3LYP ground state calculations are the closest to the $\mathrm{CC} 2$ reference. However, this property is only overestimated by $5 \mathrm{meV}$ for $4 \mathrm{CzIPN}$, whereas for $3 \mathrm{CzClIPN}$ the overestimation is $19 \mathrm{meV}$. Therefore, the difference in $\Delta E_{\mathrm{ST}}$ is much too low with $5 \mathrm{meV}$. Employing sTDA leads to an increase of the $S_{1}$ energies, but hardly affects the $T_{1}$ energies. Owing to this, $\Delta E_{\mathrm{ST}}$ increases and also their difference becomes slightly larger with $9 \mathrm{meV}$. Analyzing the results from STD-DFT and sTDA calculations of $\Delta E_{\mathrm{ST}}$ based on ground state calculations with the rangeseparated hybrid functional CAM-B3LYP, similar trends are found. However, the values for $\Delta E_{\mathrm{ST}}$ are even higher than their B3LYP counterparts. Nonetheless, the sTDDFT calculations with CAM-B3LYP functional yield a difference of $28 \mathrm{meV}$ for $\Delta E_{\mathrm{ST}}$ between the two compounds, which is the closest to the $\mathrm{CC} 2$ reference and experiment from all DFT-based calculations. Therefore, this approach appears promising for the investigation of trends in this property, whereas sTD-DFT calculations based on B3LYP ground state calculations might underestimate such changes. Overall, the semiempirical sTDDFT and sTDA methods yield values for $\Delta E_{\mathrm{ST}}$ that are much closer to the Post-Hartree-Fock calculations than the ones from regular TD-DFT and TDA calculations (see $\mathrm{SI})$.

Insights into the nature of the $S_{1}$ and $T_{1}$ states can be obtained by visualizing the natural transition orbitals (NTOs), see figure 4. NTOs are compact orbital representations for the transition density resulting in a minimum number of electron-hole excitations. ${ }^{42}$ For all investigated electronic transitions, one pair of NTOs is dominant. These orbitals demonstrate that the $S_{1}$ and $T_{1}$ states are rather similar and there are also only small differences between the two compounds. The excited electron is mainly localized at the two cyano groups and the central benzene moiety. The hole is also found at the latter part, but it is also present at the carbazole units. It appears that the charge transfer character is more pronounced for $3 \mathrm{CzClIPN}$ than for $4 \mathrm{CzIPN}$.

To quantifiy these findings, we performed wavefunction analysis and report the outcome for unscaled CC2 in the following. For this purpose, the molecules are divided into three fragments: the first one consists of the carbazole units, the second one of the benzene unit plus the chlorine atom in case of $3 \mathrm{CzClIPN}$, and the third one of the cyano groups. Based on this fragmentation, we obtain similar values from wavefunction analysis for the $S_{1}$ and $T_{1}$ states of each compound. The charge transfer character can be used as a quantitative descriptor. It ranges from 0 for an excitation completely localized at one fragment to 1 for the case that electron and hole are localized at different fragments. ${ }^{43}$ For $4 \mathrm{CzIPN}$, this charge transfer character is 0.78 and 0.77 for $S_{1}$ and $T_{1}$, respectively, and it increases to 0.84 and 0.83 for $3 \mathrm{CzClIPN}$. Therefore, the increase in charge transfer 
4CzIPN

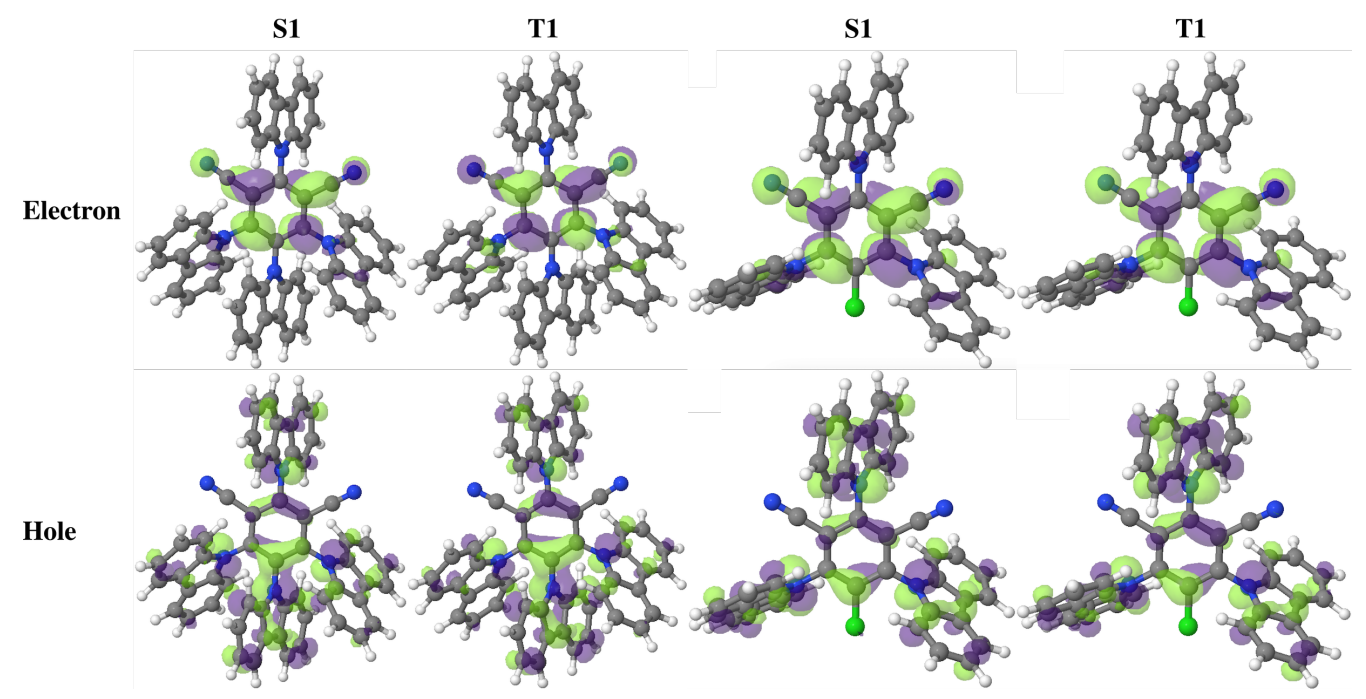

FIG. 4. Natural transition orbitals (NTO) of the first excited singlet state $\left(S_{1}\right)$ and the first triplet state $\left(T_{1}\right)$ from the CC2 calculations. For comparability, all NTOs are visualized with the same isovalue.

character leads to a decrease of $\Delta E_{\mathrm{ST}}$ and further information on the performed wavefunction analysis along with the results for $3 \mathrm{CzHIPN}$ is given in the SI.

Next, we discuss the influence of the molecular environment in our measurements. Molecules which are sufficiently separated are known to show differences and fluctuations of their emission ${ }^{44,45}$ and absorption ${ }^{46}$ spectra and lifetime. ${ }^{28}$ This is caused by static differences of the molecular environment and dynamic variations of, for example, the side group movement and alignment of the molecule. The influence of the matrix (host) and molecular arrangement on TADF properties is widely discussed in the literature. ${ }^{25,30,34,37,47-52}$ To study the non-monoexponential decay characteristics of $4 \mathrm{CzIPN}$ and $3 \mathrm{CzClIPN}$ in film, we compared neat films to PS films doped with a low $(1 \mathrm{wt} \%)$ concentration of TADF molecules. Neat films of $4 \mathrm{CzIPN}$ and $3 \mathrm{CzClIPN}$ show a strong red-shift and lower PLQY in comparison to doped PS films (see SI). This is mainly caused by selfabsorption (reabsorption within the film) and self-quenching. ${ }^{37,53}$ Polystyrene films doped with $1 \mathrm{wt} \%$ of $4 \mathrm{CzIPN}$ showed a slightly increased $\Delta E_{\mathrm{ST}}$ compared to neat films. Considering the error of $\pm 8 \mathrm{meV}, 3 \mathrm{CzClIPN}$ shows similar results as neat film or embedded in PS. This indicates, that solid state solvation effects caused by matrix polarization and static charge transfer state stabilization ${ }^{47}$ are minor when embedding $4 \mathrm{CzIPN}$ in PS, and negligible in case of $3 \mathrm{CzClIPN}$. This result is in agreement with a supplementary measurement by Olivier et al. who found similar decay characteristics of a neat $2 \mathrm{CzPN}(1,2-$ bis(carbazol-9-yl)-4,5-dicyanobenzene) film compared to a doped $98 \mathrm{wt} \%$ PS film. ${ }^{30}$ We conclude that - although spectral properties are strongly affected by the matrixthe similar stretched exponential characteristics of the transients are not only caused by static or dynamic film disorder, but by the molecules' large dihedral side group angles. This is in agreement with the work of Hasegawa et al. who found that the electronic states of $4 \mathrm{CzIPN}$ films are similar to the monomer. ${ }^{50}$

\section{CONCLUSIONS}

By investigating steady-state and time-dependent fluorescence characteristics, we elucidated the role of chlorine on the internal transition rates and singlet-triplet energy splitting $\Delta E_{\mathrm{ST}}$ of $3 \mathrm{CzClIPN}$ films compared to $4 \mathrm{CzIPN}$. Although the photoluminescence and electroluminescence spectra of both materials is similar, quantum yield and temperature-dependent delayed fluorescence are drastically influenced by the chlorine group. Photoluminescence quantum yield was reduced by factor 2 in $3 \mathrm{CzClIPN}$, due to nonradiative transitions from the excited singlet to the ground state. Reverse intersystem crossing is strongly increased in $3 \mathrm{CzClIPN}$ by factor 3-5 due to the lowered $\Delta E_{\mathrm{ST}}$. Quantum chemical calculations explained these findings by showing that chlorine increases the charge transfer character of the relevant states. This may also explain the increased photocatalytic efficiency of $3 \mathrm{CzClIPN}$ in oxidations which was recently demonstrated. ${ }^{9}$ For potential applications of $3 \mathrm{CzClIPN}$ in OLED devices, the nonradiative losses are a disadvantage. Our findings underline that precise knowledge of the excited states alone is insufficient in predicting the performance of a TADF molecule for potential applications in photocatalysis and OLED devices. To gain the necessary additional information about internal transition rates, we introduced a robust global evaluation 
method for transient fluorescence data which outputs all parameters of the specified TADF model in one single optimization step. By comparing neat films to diluted polystyrene films, we concluded that the often observed stretched exponential transient characteristics of TADF materials are a feature of large dihedral side group angles in condensed phase.

\section{METHODS}

All decay data sets and fit functions were normalized in order to reduce optimization parameters. The algorithm then minimized the global difference between temperature-dependent data and the solution of the temperature-dependent rate equations. The global difference vector was weighted with a three part step function, emphasizing the $1-5 \mu \mathrm{s}$ part of the decay, where $\mathrm{DF}$ is measured with the best signal-to-noise ratio. All fit parameters $\left(N_{1}, N_{2}, k_{\mathrm{F}}^{1}, k_{\mathrm{F}}^{2}, k_{\mathrm{ISC}}, k_{\mathrm{A}}, \Delta E_{\mathrm{ST}}\right)$ are global. TADF and TADF:polystyrene (PS) films were prepared by spincoating. Silicon substrates with $100 \mathrm{~nm}$ of thermally grown oxide were used. Substrates and glassware were cleaned by annealing at $450^{\circ} \mathrm{C}$ for two hours in a laboratory oven. PS with a molar mass of $20 \mathrm{~kg} \mathrm{~mol}^{-1}$ was doped with $1 \mathrm{wt} \%$ of $4 \mathrm{CzIPN}$ and $3 \mathrm{CzClIPN}$, respectively, at a total concentration of $10 \mathrm{mg} \mathrm{ml}^{-1}$. For neat dye films, $0.1 \mathrm{mg} \mathrm{ml}^{-1}$ of dye in toluene was used. The samples were measured with a homebuilt laser scan confocal microscope in a cryostate (Janis, ST500) with a Zeiss LWD $63 \times, \mathrm{NA}=0.75$ objective. Fluorescence decay was acquired with time-correlated single photon counting (TCSPC) using a Perkin Elmer avalanche photodiode (APD) and a PicoHarp300 TCSPC module (PicoQuant). The PicoHarp300 was set to the maximum time window of $33.55 \mu$ s with a time resolution of $0.512 \mathrm{~ns}$ per channel. Data was logarithmically binned and the background level substracted. The samples were excited with a $465 \mathrm{~nm}$ pulsed diode laser (PicoQuant, $\tau_{\text {IRF }}<1 \mathrm{~ns}$ ) at a repetition rate of $25 \mathrm{kHz}$. Fluorescence was detected with a $500 \mathrm{~nm}$ longpass filter. Each decay curve was measured by integrating for 15 minutes. The dark count rate of the APD was $50 \mathrm{cts}$ (counts per second). The objective was defocussed until the fluorescence signal was below 1000 cts on the APD to ensure falling below the general $5 \%$ limit of the TCSPC technique (signal photons to laser pulses per time interval). ${ }^{54}$ This condition avoids pile-up effects caused by the total dead time of the TCSPC system. Although the dark count to signal ratio suggests a signal to noise ratio of only $10: 1$, the effective ratio is better than 10000:1 because $1000 \mathrm{cts}$ of signal refer to the decay occurring within few microseconds while the dark count rate refers to one second. We want to emphasize that measuring time-dependent fluorescence in both, nanosecond and microsecond (or millisecond) time regimes with equal experimental parameters is challenging and demands ensuring appropriate equilibrium conditions of optical excitation and decay.
Measuring at non-equilibrium conditions leads to wrong results and conclusions. This is especially the case at low temperatures, materials with long DF lifetimes and when measuring PF and DF separately with different excitation sources (or laser repetition rates) on different time scales.

\section{ACKNOWLEDGMENTS}

C. W. acknowledges funding by the German Research Foundation (DFG) via a return fellowship (reference number: WI 4853/2-1). The quantum chemical calculations were performed on ressources provided by the Leipzig University Computing Centre and by the Paderborn Center for Parallel Computing. C. W. also thanks Stefan Zahn, Bernd Abel, and Jörg Matysik for discussions and support.

\section{CORRESPONDING AUTHOR}

Email: deibel@physik.tu-chemnitz.de

\section{NOTES}

The authors declare no competing financial interest.

\section{SUPPLEMENTARY INFORMATION}

Inverse Laplace fit results, additional rate model fit results, global rate fit results, $k_{\mathrm{RISC}}(T)$ plots, simulation results, UV-Vis spectra, quantum yield, streak measurements, quantum chemical calculations 


\section{REFERENCES}

${ }^{1}$ Hung, L.; Chen, C. Recent Progress of Molecular Organic Electroluminescent Materials and Devices. Mater. Sci. Eng. R Rep 2002, 39, 143-222.

2 Reineke, S.; Lindner, F.; Schwartz, G.; Seidler, N.; Walzer, K.; Lüssem, B.; Leo, K. White Organic Lightemitting Diodes with Fluorescent Tube Efficiency. Nature 2009, 459, 234.

${ }^{3}$ Sun, Y.; Giebink, N. C.; Kanno, H.; Ma, B.; Thompson, M. E.; Forrest, S. R. Management of Singlet and Triplet Excitons for Efficient White Organic Lightemitting Devices. Nature 2006, 440, 908.

${ }^{4}$ Zeitler, K. Photoredox Catalysis with Visible Light. Angew. Chem. Int. Ed. 2009, 48, 9785-9789.

${ }^{5}$ Adachi, C.; Baldo, M. A.; Thompson, M. E.; Forrest, S. R. Nearly $100 \%$ Internal Phosphorescence Efficiency in an Organic Light-emitting Device. J. Appl. Phys. 2001, 90, 5048-5051.

${ }^{6}$ Uoyama, H.; Goushi, K.; Shizu, K.; Nomura, H.; Adachi, C. Highly Efficient Organic Light-emitting Diodes from Delayed Fluorescence. Nature 2012, 492, 234.

7 Onsager, L. Reciprocal Relations in Irreversible Processes. I. Phys. Rev. 1931, 37, 405.

${ }^{8}$ Hirata, S.; Sakai, Y.; Masui, K.; Tanaka, H.; Lee, S. Y.; Nomura, H.; Nakamura, N.; Yasumatsu, M.; Nakanotani, H.; Zhang, Q., et al. Highly Efficient Blue Electroluminescence Based on Thermally Activated Delayed Fluorescence. Nat. Mater. 2015, 14, 330.

9 Speckmeier, E.; Fischer, T. G.; Zeitler, K. A Toolbox Approach To Construct Broadly Applicable Metal-Free Catalysts for Photoredox Chemistry: Deliberate Tuning of Redox Potentials and Importance of Halogens in DonorAcceptor Cyanoarenes. J. Am. Chem. Soc. 2018, 140, 15353-15365.

10 Lowry, M. S.; Goldsmith, J. I.; Slinker, J. D.; Rohl, R.; Pascal, R. A.; Malliaras, G. G.; Bernhard, S. Single-layer Electroluminescent Devices and Photoinduced Hydrogen Production from an Ionic Iridium (III) Complex. Chem. Mater. 2005, 17, 5712-5719.

11 Xiang, Y.; Zhao, Y.; Xu, N.; Gong, S.; Ni, F.; Wu, K.; Luo, J.; Xie, G.; Lu, Z.-H.; Yang, C. Halogen-induced Internal Heavy-atom Effect Shortening the Emissive Lifetime and Improving the Fluorescence Efficiency of Thermally Activated Delayed Fluorescence Emitters. J. Mater. Chem. C. 2017, 5, 12204-12210.

${ }^{12}$ Kretzschmar, A.; Patze, C.; Schwaebel, S. T.; Bunz, U. H. Development of Thermally Activated Delayed Fluorescence Materials with Shortened Emissive Lifetimes. J. Org. Chem. 2015, 80, 9126-9131.

13 Dias, F. B.; Bourdakos, K. N.; Jankus, V.; Moss, K. C.; Kamtekar, K. T.; Bhalla, V.; Santos, J.; Bryce, M. R.; Monkman, A. P. Triplet Harvesting with 100\% Efficiency by Way of Thermally Activated Delayed Fluorescence in Charge Transfer OLED Emitters. Adv. Mater. 2013, 25, 3707-3714.

14 Dias, F. B.; Penfold, T. J.; Monkman, A. P. Photophysics of Thermally Activated Delayed Fluorescence Molecules. Methods Appl. Fluoresc. 2017, 5, 012001.
15 Zhang, Q.; Li, B.; Huang, S.; Nomura, H.; Tanaka, H.; Adachi, C. Efficient Blue Organic Light-emitting Diodes Employing Thermally Activated Delayed Fluorescence. Nat. Photonics 2014, 8, 326.

16 Gibson, J.; Monkman, A. P.; Penfold, T. J. The Importance of Vibronic Coupling for Efficient Reverse Intersystem Crossing in Thermally Activated Delayed Fluorescence Molecules. ChemPhysChem 2016, 17, 2956-2961.

17 Zhang, Q.; Li, J.; Shizu, K.; Huang, S.; Hirata, S.; Miyazaki, H.; Adachi, C. Design of Efficient Thermally Activated Delayed Fluorescence Materials for Pure Blue Organic Light Emitting Diodes. J. Am. Chem. Soc. 2012, 134, 14706-14709.

18 Berberan-Santos, M. N.; Garcia, J. M. Unusually Strong Delayed Fluorescence of C70. J. Am. Chem. Soc. 1996, 118, 9391-9394.

19 Baleizão, C.; Berberan-Santos, M. N. Thermally Activated Delayed Fluorescence as a Cycling Process between Excited Singlet and Triplet States: Application to the Fullerenes. J. Chem. Phys. 2007, 126, 204510.

20 Penfold, T.; Dias, F.; Monkman, A. The Theory of Thermally Activated Delayed Fluorescence for Organic Light Emitting Diodes. Chem Comm 2018, 54, 3926-3935.

${ }^{21}$ Scholz, R.; Kleine, P.; Lygaitis, R.; Popp, L.; Lenk, S.; Etherington, M. K.; Monkman, A. P.; Reineke, S. Investigation of Thermally Activated Delayed Fluorescence from a Donor-Acceptor Compound with Time-Resolved Fluorescence and Density Functional Theory Applying an Optimally Tuned Range-Separated Hybrid Functional. J. Phys. Chem. A 2020, 124, 1535-1553.

22 Haase, N.; Danos, A.; Pflumm, C.; Morherr, A.; Stachelek, P.; Mekic, A.; Brütting, W.; Monkman, A. P. Kinetic Modeling of Transient Photoluminescence from Thermally Activated Delayed Fluorescence. J. Phys. Chem. C 2018, 122, 29173-29179.

23 Reichert, S.; Flemming, J.; An, Q.; Vaynzof, Y.; Pietschmann, J.-F.; Deibel, C. Ionic-Defect Distribution Revealed by Improved Evaluation of Deep-Level Transient Spectroscopy on Perovskite Solar Cells. Phys. Rev. Appl. 2020, 13, 034018.

24 Lakowicz, J. R. Principles of Fluorescence Spectroscopy; Springer Science \& Business Media, 2013.

${ }^{25}$ Hosokai, T.; Nakanotani, H.; Santou, S.; Noda, H.; Nakayama, Y.; Adachi, C. TADF Activation by Solvent Freezing: The Role of Nonradiative Triplet Decay and Spin-orbit Coupling in Carbazole Benzonitrile Derivatives. Synth. Met. 2019, 252, 62-68.

${ }^{26}$ Kobayashi, T.; Niwa, A.; Takaki, K.; Haseyama, S.; Nagase, T.; Goushi, K.; Adachi, C.; Naito, H. Contributions of a Higher Triplet Excited State to the Emission Properties of a Thermally Activated Delayed Fluorescence Emitter. Phys. Rev. Appl. 2017, 7, 034002.

27 Noriega, R.; Barnard, E. S.; Ursprung, B.; Cotts, B. L.; Penwell, S. B.; Schuck, P. J.; Ginsberg, N. S. Uncovering Single-molecule Photophysical Heterogeneity of Bright, Thermally Activated Delayed Fluorescence Emitters Dispersed in Glassy Hosts. J. Am. Chem. Soc. 2016, 138, 
13551-13560.

28 Börner, R.; Kowerko, D.; Krause, S.; Borczyskowski, C. v.; Hübner, C. G. Efficient Simultaneous Fluorescence Orientation, Spectrum, and Lifetime Detection for Single Molecule Dynamics. J. Chem. Phys. 2012, 137, 164202.

${ }^{29}$ Yurash, B.; Nakanotani, H.; Olivier, Y.; Beljonne, D.; Adachi, C.; Nguyen, T.-Q. Photoluminescence Quenching Probes Spin Conversion and Exciton Dynamics in Thermally Activated Delayed Fluorescence Materials. Adv. Mater. 2019, 31, 1804490.

30 Olivier, Y.; Yurash, B.; Muccioli, L.; D'Avino, G.; Mikhnenko, O.; Sancho-Garcia, J.-C.; Adachi, C.; Nguyen, T.-Q.; Beljonne, D. Nature of the Singlet and Triplet Excitations Mediating Thermally Activated Delayed Fluorescence. Phys. Rev. Mater. 2017, 1, 075602.

31 Noda, H.; Chen, X.-K.; Nakanotani, H.; Hosokai, T.; Miyajima, M.; Notsuka, N.; Kashima, Y.; Brédas, J.L.; Adachi, C. Critical Role of Intermediate Electronic States for Spin-flip Processes in Charge-transfer-type Organic Molecules with Multiple Donors and Acceptors. Nat. Mater. 2019, 18, 1084-1090.

32 Menke, S. M.; Holmes, R. J. Exciton Transport in an Organic Semiconductor Exhibiting Thermally Activated Delayed Fluorescence. J. Phys. Chem. C 2016, 120, 85028508 .

33 Niwa, A.; Kobayashi, T.; Nagase, T.; Goushi, K.; Adachi, C.; Naito, H. Temperature Dependence of Photoluminescence Properties in a Thermally Activated Delayed Fluorescence Emitter. Appl. Phys. Lett. 2014, 104, 79_1.

34 Ishimatsu, R.; Matsunami, S.; Shizu, K.; Adachi, C.; Nakano, K.; Imato, T. Solvent Effect on Thermally Activated Delayed Fluorescence by 1, 2, 3, 5-tetrakis (carbazol9-yl)-4, 6-dicyanobenzene. J. Phys. Chem. A 2013, 117, $5607-5612$.

${ }^{35}$ Kobayashi, T.; Kawate, D.; Niwa, A.; Nagase, T.; Goushi, K.; Adachi, C.; Naito, H. Intersystem Crossing Rate in Thermally Activated Delayed Fluorescence Emitters. Phys. Status Solidi A 2019,

${ }^{36}$ Einzinger, M.; Zhu, T.; de Silva, P.; Belger, C.; Swager, T. M.; Van Voorhis, T.; Baldo, M. A. Shorter Exciton Lifetimes via an External Heavy-Atom Effect: Alleviating the Effects of Bimolecular Processes in Organic Light-Emitting Diodes. Adv. Mater. 2017, 29, 1701987.

37 Kim, H. S.; Park, S.-R.; Suh, M. C. Concentration Quenching Behavior of Thermally Activated Delayed Fluorescence in a Solid Film. J. Phys. Chem. C 2017, 121, 13986-13997.

38 Penfold, T. J. On Predicting the Excited-State Properties of Thermally Activated Delayed Fluorescence Emitters. J. Phys. Chem. C 2015, 119, 13535-13544.

39 Theoretical Predication for Transition Energies of Thermally Activated Delayed Fluorescence Molecules. Chin. Chem. Lett. 2016, 27, 1445-1452.

40 Sun, H.; Zhong, C.; Brédas, J.-L. Reliable Prediction with Tuned Range-Separated Functionals of the Singlet-Triplet Gap in Organic Emitters for Thermally Activated Delayed Fluorescence. J. Chem. Theory Comput. 2015, 11, 38513858 .

41 Olivier, Y.; Moral, M.; Muccioli, L.; Sancho-García, J.C. Dynamic Nature of Excited States of Donor-acceptor
TADF Materials for OLEDs: How Theory can Reveal Structure-property Relationships. J. Mater . Chem. C 2017, 5, 5718-5729.

42 Martin, R. L. Natural Transition Orbitals. J. Chem. Phys. 2003, 118, 4775-4777.

43 Plasser, F.; Lischka, H. Analysis of Excitonic and Charge Transfer Interactions from Quantum Chemical Calculations. J. Chem. Theory Comput. 2012, 8, 2777-2789.

44 Ambrose, W.; Moerner, W. Fluorescence Spectroscopy and Spectral Diffusion of Single Impurity Molecules in a Crystal. Nature 1991, 349, 225.

45 Krause, S.; Kowerko, D.; Börner, R.; Hübner, C. G.; von Borczyskowski, C. Spectral Diffusion of Single Molecules in a Hierarchical Energy Landscape. ChemPhysChem 2011, 12, 303-312.

46 Streiter, M.; Krause, S.; von Borczyskowski, C.; Deibel, C. Dynamics of Single-molecule Stokes Shifts: Influence of Conformation and Environment. J. Phys. Chem. Lett. 2016, 7, 4281-4284.

47 Northey, T.; Stacey, J.; Penfold, T. The Role of Solid State Solvation on the Charge Transfer State of a Thermally Activated Delayed Fluorescence Emitter. J. Mater. Chem. C 2017, 5, 11001-11009.

48 Cho, Y. J.; Yook, K. S.; Lee, J. Y. High Efficiency in a Solution-processed Thermally Activated Delayedfluorescence Device Using a Delayed-fluorescence Emitting Material with Improved Solubility. Adv. Mater. 2014, 26, 6642-6646

${ }^{49}$ Mamada, M.; Inada, K.; Komino, T.; Potscavage Jr, W. J.; Nakanotani, H.; Adachi, C. Highly Efficient Thermally Activated Delayed Fluorescence from an Excited-state Intramolecular Proton Transfer System. ACS Cent. Sci. 2017, 3, 769-777.

50 Hasegawa, Y.; Yamada, Y.; Sasaki, M.; Hosokai, T.; Nakanotani, H.; Adachi, C. Well-ordered 4CzIPN ((4s, 6s)2, 4, 5, 6-Tetra (9-H-carbazol-9-yl) isophthalonitrile) Layers: Molecular Orientation, Electronic Structure, and Angular Distribution of Photoluminescence. J. Phys. Chem. Lett. 2018, 9, 863-867.

${ }^{51}$ Cucchi, M.; Matulaitis, T.; Kukhta, N. A.; Grazulevicius, J. V.; Reineke, S.; Scholz, R. Influence of the Dielectric Constant Around an Emitter on its Delayed Fluorescence. Phys. Rev. Appl. 2019, 12, 044021.

52 Takeda, H.; Takeda, M.; Yoshioka, H.; Minamide, H.; Oki, Y.; Adachi, C. Fluorescence Lifetime Elongation of Thermally Activated Delayed Fluorescence 4CzIPN Molecules with Encapsulation into Zeolitic Imidazole Frameworks ZIF-11. Opt. Mater. Express 2019, 9, 11501160.

53 Ahn, T.-S.; Al-Kaysi, R. O.; Müller, A. M.; Wentz, K. M.; Bardeen, C. J. Self-absorption Correction for Solid-state Photoluminescence Quantum Yields Obtained from Integrating Sphere Measurements. Rev. Sci. Instrum. 2007, 78, 086105.

54 Becker, W. The bh TCSPC handbook; Becker \& Hickl, 2014. 


\title{
Supporting Information
}

Impact of chlorine on the internal transition rates and excited states of the thermally delayed activated fluorescence molecule $3 \mathrm{CzClIPN}$

\author{
Martin Streiter, ${ }^{1}$ Tillmann Fischer, ${ }^{2}$ Christian Wiebeler, ${ }^{3,4}$ Sebastian \\ Reichert, ${ }^{1}$ Jörn Langenickel, ${ }^{5}$ Kirsten Zeitler, ${ }^{2}$ and Carsten Deibel ${ }^{1}$ \\ ${ }^{1}$ Institut für Physik, Technische Universität Chemnitz, 09126 Chemnitz, Germany \\ ${ }^{2}$ Institut für Organische Chemie, Universität Leipzig, 04103 Leipzig, Germany \\ ${ }^{3}$ Institut für Analytische Chemie, Universität Leipzig, 04103 Leipzig, Germany \\ ${ }^{4}$ Leibniz-Institut für Oberflächenmodifizierung (IOM), 04318 Leipzig, Germany \\ ${ }^{5}$ Zentrum für Mikrotechnologien, Technische Universität Chemnitz, 09126 Chemnitz, Germany
}




\section{EQUATIONS}

Effective rate $k_{\mathrm{DF}}$ :

$$
\left\langle k_{\mathrm{DF}}\right\rangle=\frac{A_{1} k_{\mathrm{DF} 1}^{-1}+A_{2} k_{\mathrm{DF} 1}^{-1}}{A_{1} k_{\mathrm{DF} 1}^{-2}+A_{2} k_{\mathrm{DF} 2}^{-2}}
$$

Effective photoluminescence rate determined with amplitude-weighted rates $k_{\mathrm{F}}$ :

$$
\left\langle k_{\mathrm{F}}\right\rangle=\frac{A_{1} k_{\mathrm{F} 1}^{-1}+A_{2} k_{\mathrm{F} 1}^{-1}}{A_{1} k_{\mathrm{F} 1}^{-2}+A_{2} k_{\mathrm{F} 2}^{-2}}
$$

Effective photoluminescence lifetime determined with the two main amplitude-weighted exponential rates from inverse Laplace transform:

$$
\left\langle\tau_{\mathrm{PL}}\right\rangle=\frac{A_{1} k_{\mathrm{F} 1}^{-2}+A_{2} k_{\mathrm{F} 2}^{-2}}{A_{1} k_{\mathrm{F} 1}^{-1}+A_{2} k_{\mathrm{F} 1}^{-1}}
$$

\section{LAPLACE TRANSFORM FIT}

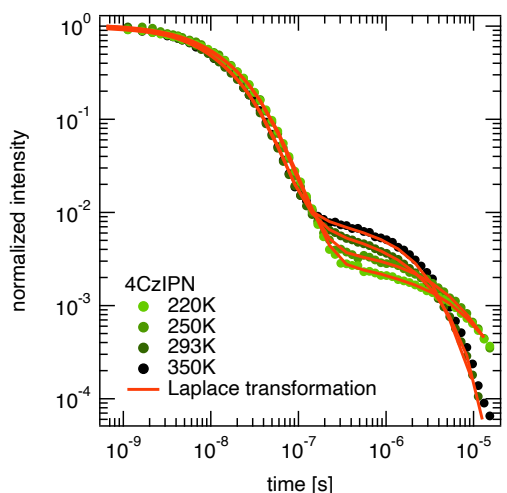

(a) $4 \mathrm{CzIPN}$

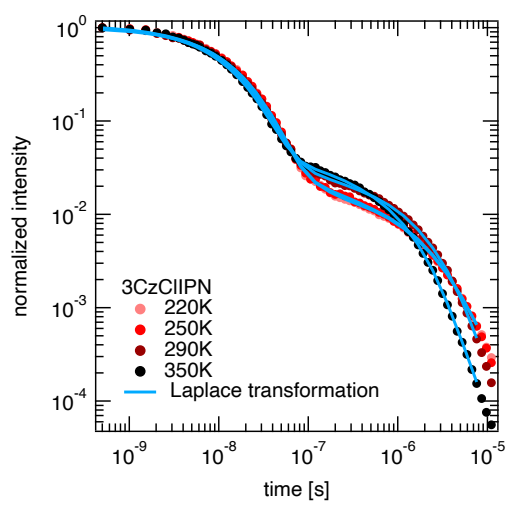

(c) $3 \mathrm{CzClIPN}$

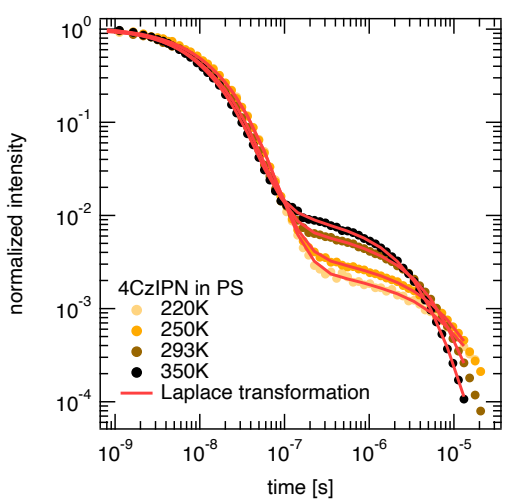

(b) $4 \mathrm{CzIPN}$ in PS

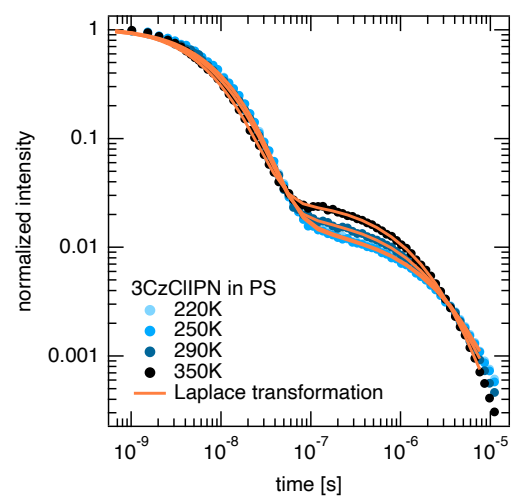

(d) $3 \mathrm{CzClIPN}$ in PS

FIG. 1: Laplace transform fits 


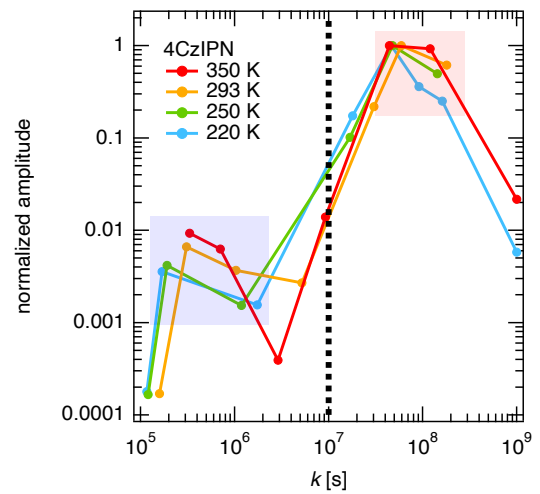

(a) $4 \mathrm{CzIPN}$

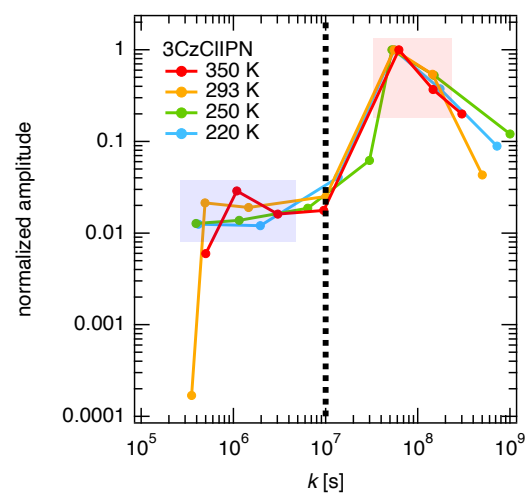

(c) $3 \mathrm{CzClIPN}$

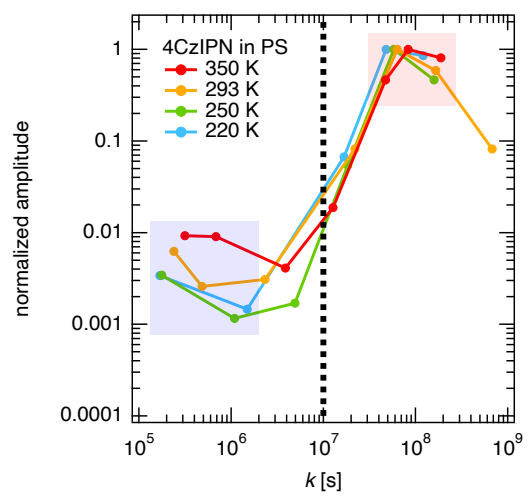

(b) $4 \mathrm{CzIPN}$ in PS

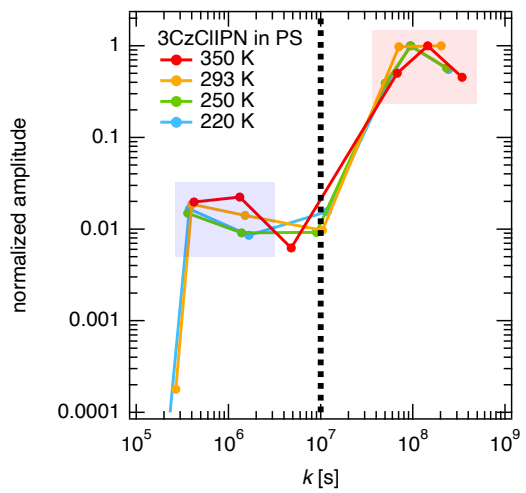

(d) $3 \mathrm{CzClIPN}$ in PS

FIG. 2: Laplace rates and amplitudes

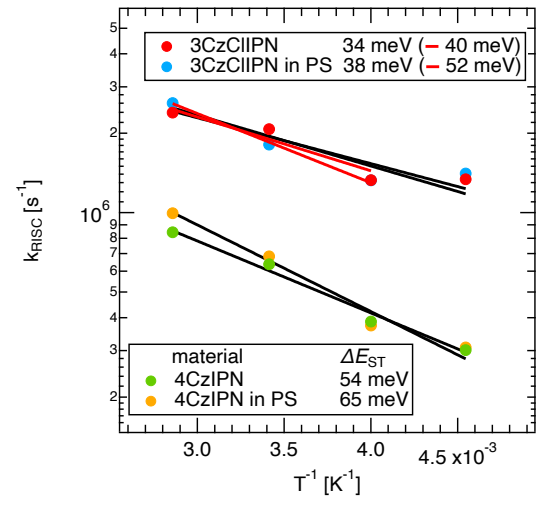

(a)

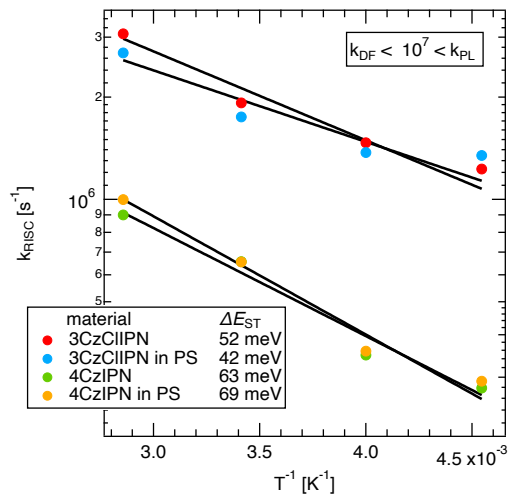

(b)

FIG. 3: Arrhenius plots of Laplace analysis 


\section{ALTERNATIVE RATE DIFFERENTIAL EQUATION FITS}

It is possible to achieve precise fits which fit the stretched exponential part of the delayed fluorescence by summing up two singlet states $S_{1}(t)+S_{2}(t)$. Both $S(t)$ functions are the solution of the follwing coupled differential equation system with individual depopulation rates $k_{\mathrm{F}}$, reverse intersystem crossing rates $k_{\mathrm{RISC}}$ and one shared intersystem crossing rate $k_{\mathrm{ISC}}$ :

$$
\begin{aligned}
& \dot{S}_{1}(t)=-\left(k_{\mathrm{F} 1}+k_{\mathrm{ISC}}\right) S_{1}(t)+k_{\mathrm{RISC} 1} T_{1}(t) \\
& \dot{T}_{1}(t)=k_{\mathrm{ISC}} S_{1}(t)-k_{\mathrm{RISC} 1} T_{1}(t) \\
& \dot{S}_{2}(t)=-\left(k_{\mathrm{F} 2}+k_{\mathrm{ISC}}\right) S_{2}(t)+k_{\mathrm{RISC} 2} T_{2}(t) \\
& \dot{T}_{2}(t)=k_{\mathrm{ISC}} S_{2}(t)-k_{\mathrm{RISC} 2} T_{2}(t) .
\end{aligned}
$$

\section{A. Fits}

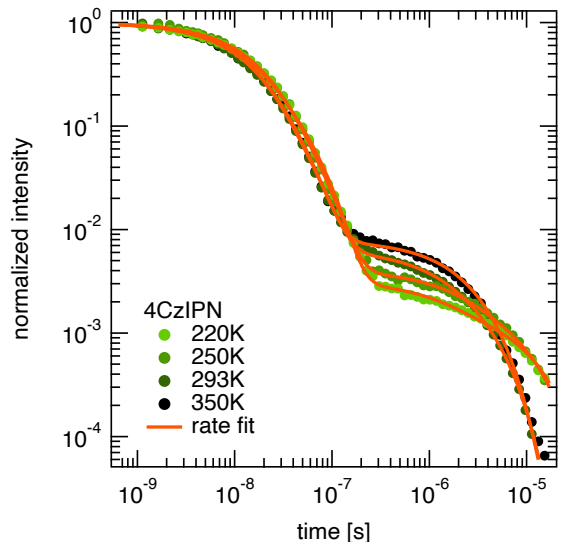

(a) $4 \mathrm{CzIPN}$

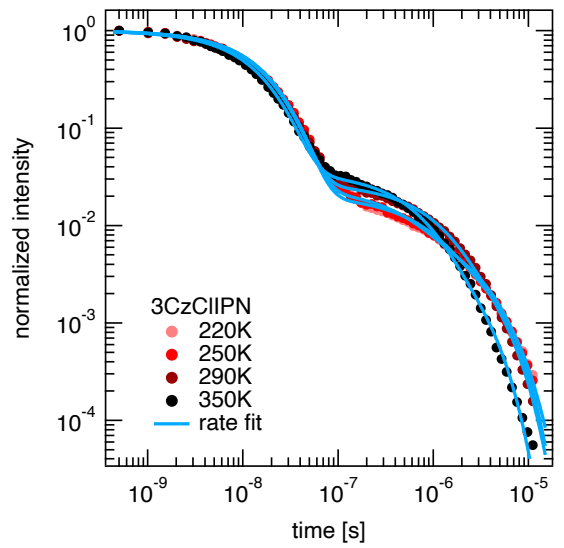

(c) $3 \mathrm{CzClIPN}$

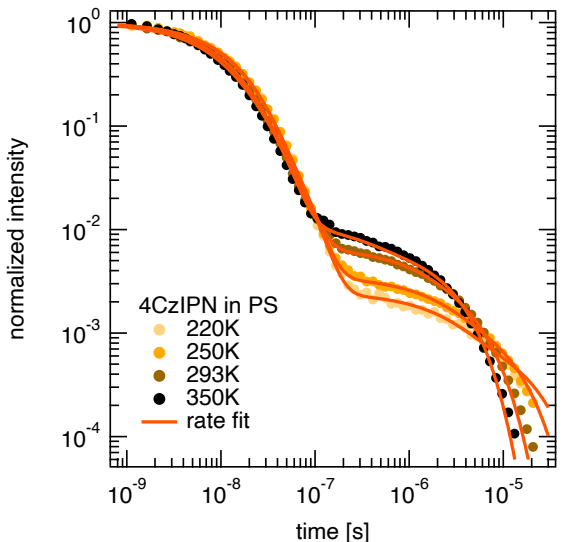

(b) 4 CzIPN in PS

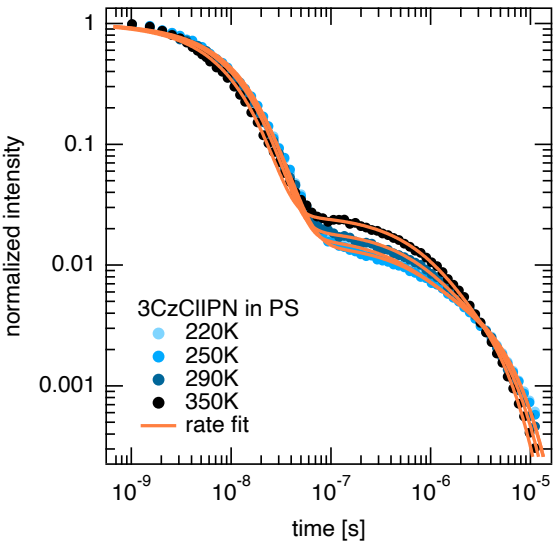

(d) $3 \mathrm{CzClIPN}$ in PS

FIG. 4: Distribution of $k_{\text {RISC }}$ 


\section{B. Rate analysis}

Assuming two different $\Delta E_{\mathrm{ST}}$ was suggested by Kobayashi et al. for neat $4 \mathrm{CzIPN}$ films. ${ }^{1}$ However, it is not clear if interpreting the Arrenhius plot is useful for two different $k_{\mathrm{RISC}}$ (or the corresponding weighted Arithmetic or geometric means as shown in figures B a-d) because they are not connected. Therefore, the deviation between both $k_{\text {RISC }}$ looks differently for each sample, although all fits have a similar quality. This also makes treating the approach as a global fit difficult. Interpreting the temperature-dependent $k_{\mathrm{RISC}}^{1}$ and $k_{\mathrm{RISC}}^{2}$ individually leads to values which are not plausible. The geometric mean deviates from the values presented in the main paper without a clear trend. All Arrenhius fits with $k_{\mathrm{RISC}}=k_{\mathrm{A}} \exp \left(-\Delta E_{\mathrm{ST}} / k_{\mathrm{B}} T\right)$ are summarized in table $\mathrm{I}$.

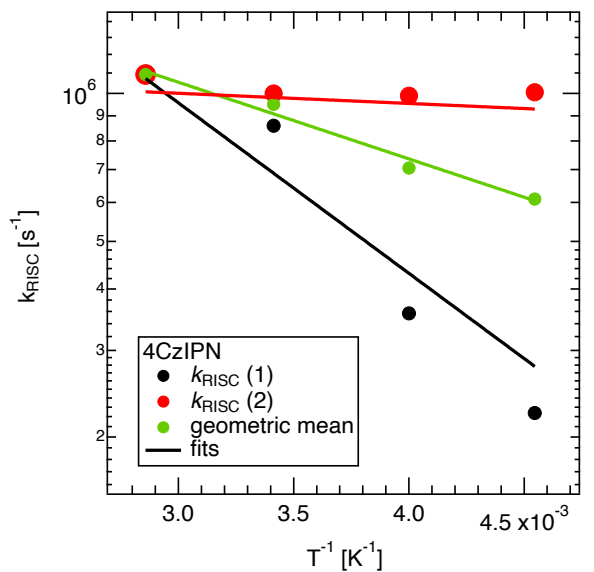

(a) $4 \mathrm{CzIPN}$

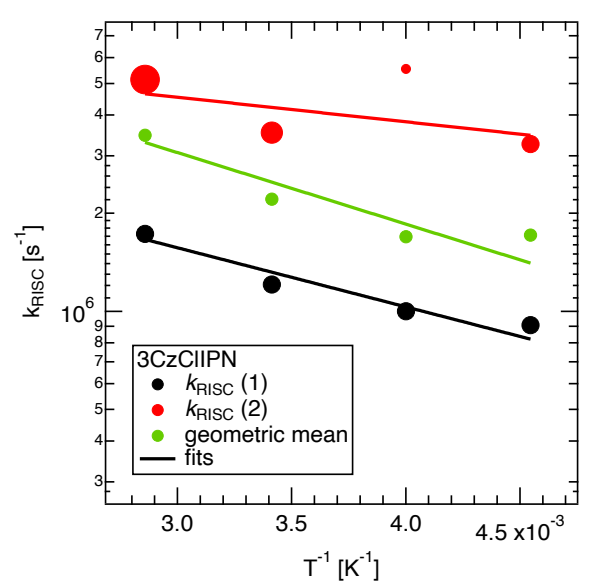

(c) $3 \mathrm{CzClIPN}$

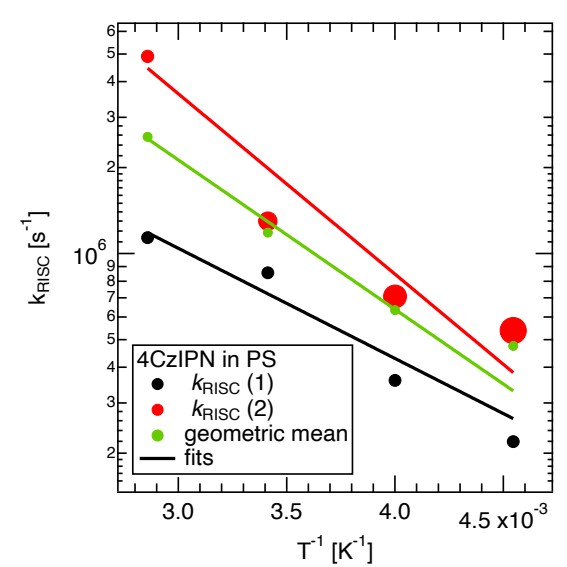

(b) $4 \mathrm{CzIPN}$ in PS

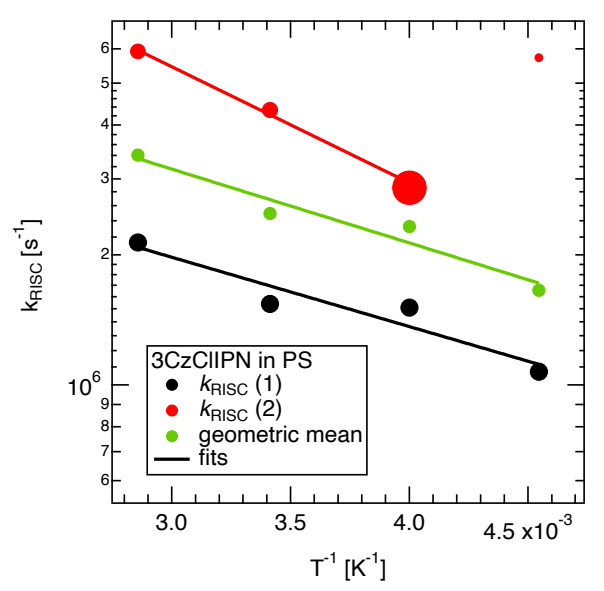

(d) $3 \mathrm{CzClIPN}$ in PS

FIG. 5: Weighted (dot size) $k_{\text {RISC }}$ rates from rate fits.

\begin{tabular}{|c|c|c|c|c|c|}
\hline & & $\begin{array}{r}4 \mathrm{CzIPN} \\
\text { neat film }\end{array}$ & in PS & $\begin{array}{c}3 \mathrm{CzClIPN} \\
\text { neat film }\end{array}$ & in PS \\
\hline$\Delta E_{\mathrm{ST}}(1)$ & {$[\mathrm{meV}]$} & 69 & 76 & 36 & 32 \\
\hline$\Delta E_{\mathrm{ST}}(2)$ & {$[\mathrm{meV}]$} & 4 & 125 & 15 & 53 \\
\hline$\Delta E_{\mathrm{ST}}($ geo $)$ & {$[\mathrm{meV}]$} & 31 & 104 & 43 & 34 \\
\hline
\end{tabular}

TABLE I: Individual $\Delta E_{\mathrm{ST}}$ and weighted geometric means of the Arrenhius plots. 
IV. GLOBAL FITS

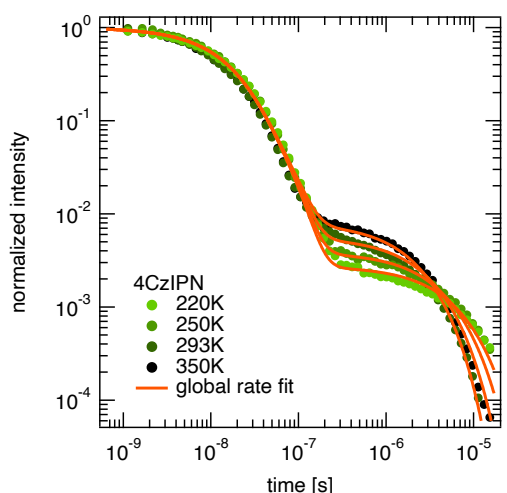

(a) $4 \mathrm{CzIPN}$

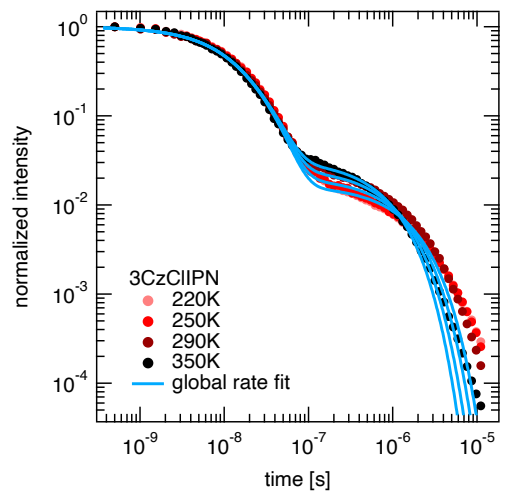

(c) $3 \mathrm{CzClIPN}$

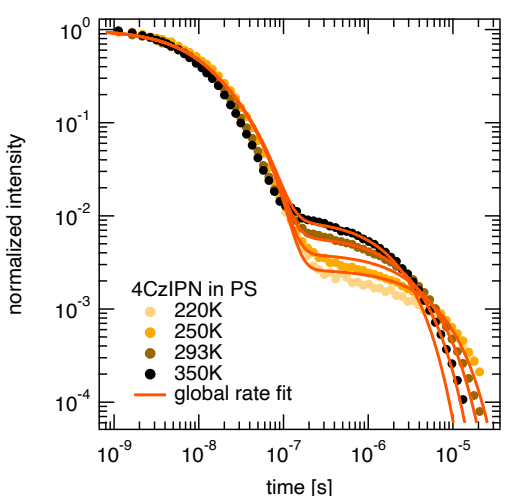

(b) 4 CzIPN in PS

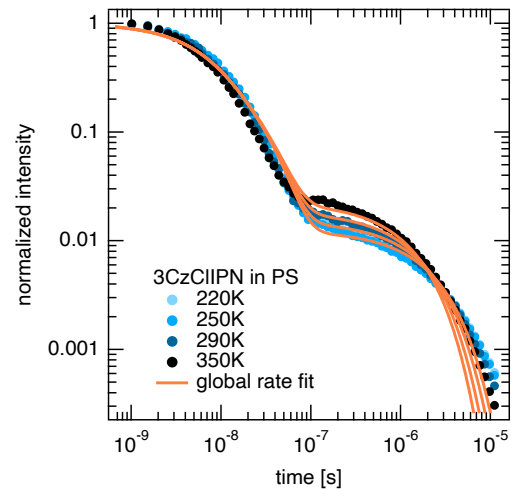

(d) $3 \mathrm{CzClIPN}$ in PS

FIG. 6: global fits

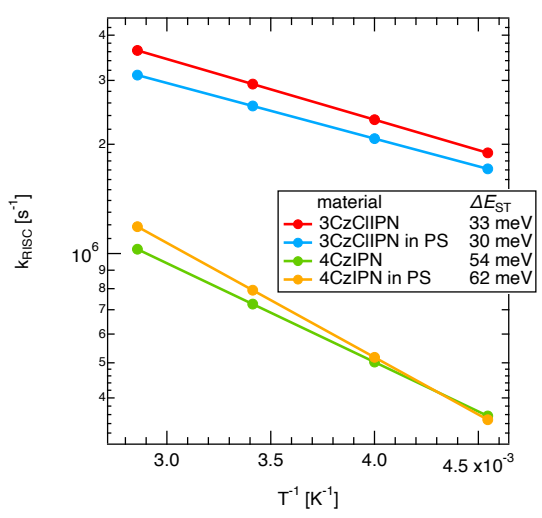

FIG. 7: Arrhenius plot of global fits 


\section{STEADY-STATE MEASUREMENTS}

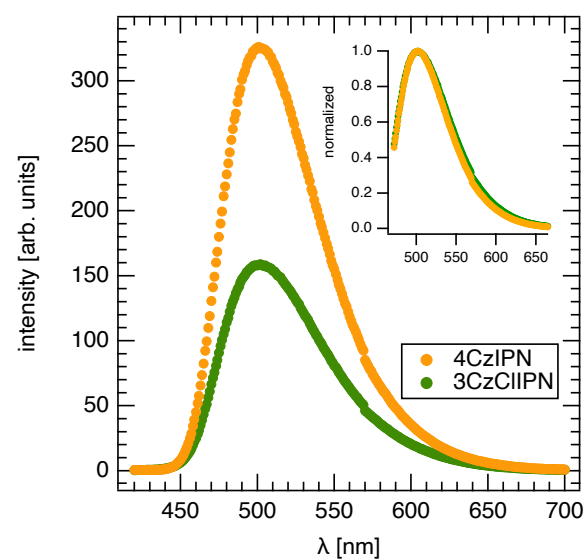

(a)Comparison of $5 \mathrm{wt} \% 4 \mathrm{CzIPN}$ and $3 \mathrm{CzClIPN}$ in CBP dissolved in toluene. CBP concentration $1 \mathrm{mg} \mathrm{ml}^{-1}$.

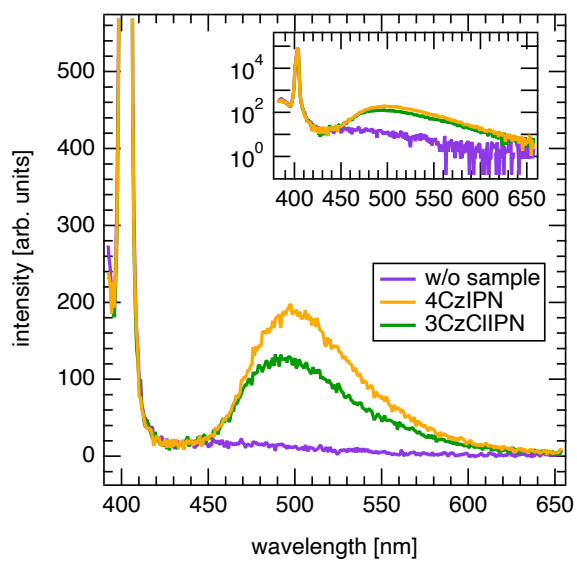

(c)Photoluminescence quantum yield (PLQY: $4 \mathrm{CzIPN} 99 \%, 3 \mathrm{CzClIPN} 54 \%$ )

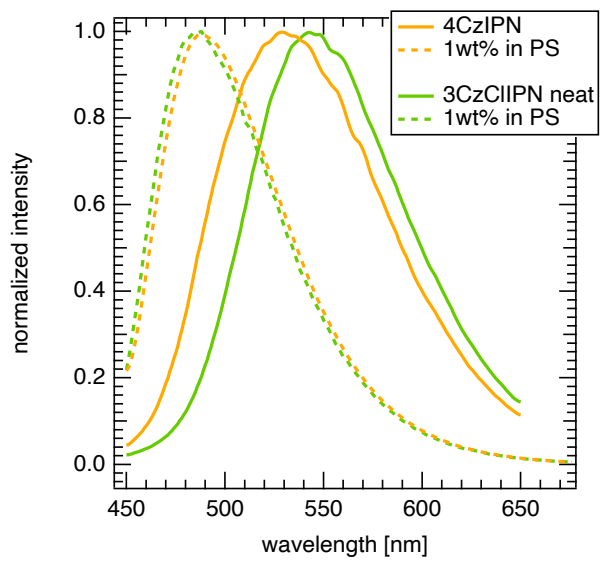

(b) Normalized steady-state PL spectra of neat and doped PS films on glass indicating red-shift for neat material due to reabsorption.

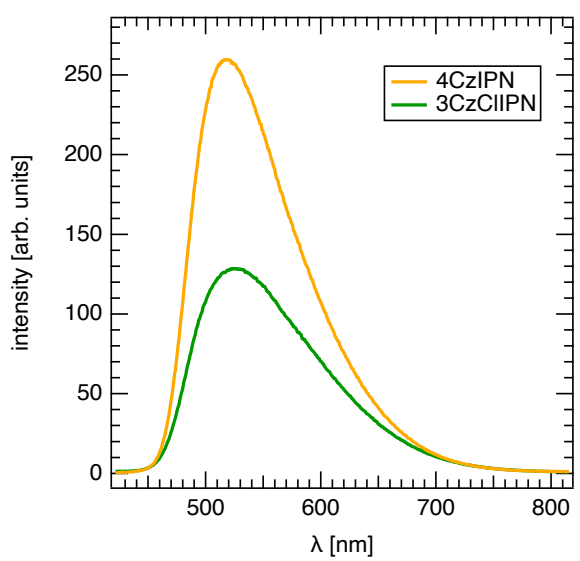

(d)Normalized electroluminescence embedded in CBP

FIG. 8: Steady-state spectra

Spectra of $4 \mathrm{CzIPN}$ and $3 \mathrm{CzClIPN}$ in solution and as films were measured with a Cary Eclipse spectrometer (Varian) and excited at $405 \mathrm{~nm}$. The absolute photoluminescence quantum yield (PLQY) was determined by measuring the spectrum (OceanOptics, QE pro - calibrated with an ORIEL 63358 tungsten lamp) of the excitation laser diode (Thorlabs, $405 \mathrm{~nm}$ ) and the $1 \mathrm{wt} \% 4 \mathrm{CzIPN}(3 \mathrm{CzClIPN})$ on glass film simultaneously. As a reference, a clean glass substrate was measured. PLQY is then calculated with (emitted PL)/(laser signal from clean glass - laser signal from TADF sample). For the measurement of electroluminescence, structured ITO substrates were spincoated with $\mathrm{ZnO}-\mathrm{NP}$ (zinc oxide nanoparticles) dissolved in ethanol $\left(50 \mathrm{mg} \mathrm{ml}^{-1}\right.$ at $2000 \mathrm{rpm}$ ) and $5 \mathrm{wt} \% \mathrm{of} 4 \mathrm{CzIPN}(3 \mathrm{CzClIPN})$ in $\mathrm{CBP}\left(4,4^{\prime}-\operatorname{Bis}\left(N\right.\right.$-carbazolyl)-1,1'-biphenyl) dissolved in toluene $\left(12 \mathrm{mg} \mathrm{ml}^{-1}\right.$ at $\left.1500 \mathrm{rpm}\right)$. These films then were subsequently evaporated with $40 \mathrm{~nm} \operatorname{HMTPD}\left(N, N, N^{\prime}, N^{\prime}\right.$-Tetrakis(3-methylphenyl)-3,3'-dimethylbenzidine), $10 \mathrm{~nm}$ molybdenum oxide and $100 \mathrm{~nm}$ aluminum. A voltage of $6 \mathrm{~V}$ was applied and the spectrum was measured with a $200 \mu \mathrm{m}$, NA 0.22 fiber which was coupled to the substrate and the spectrometer (OceanOptics, QE pro). 
VI. TIME-RESOLVED MEASUREMENTS

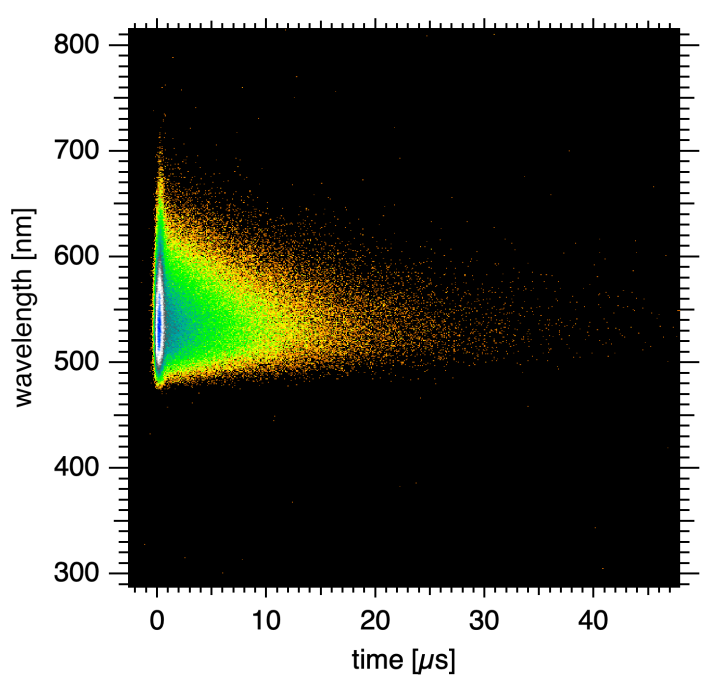

(a) $4 \mathrm{CzIPN}$ in PS

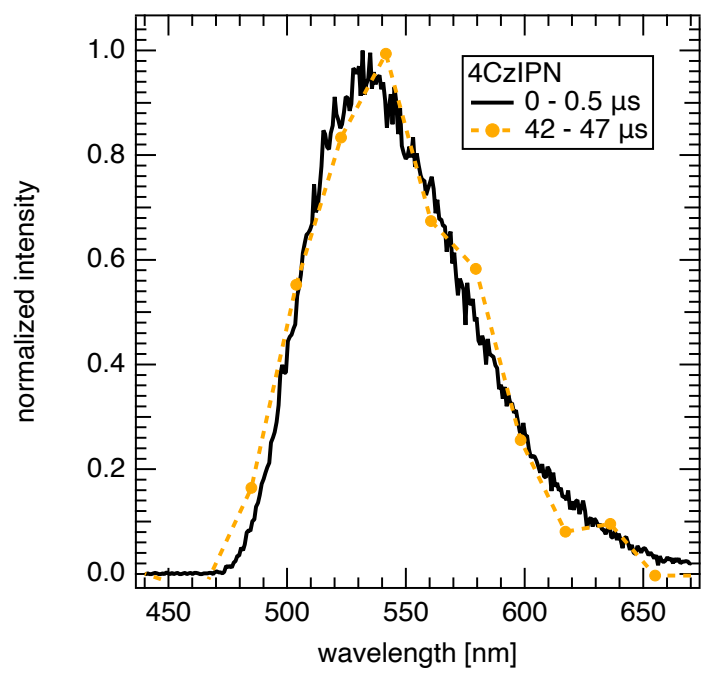

(c) 4 CzIPN in PS

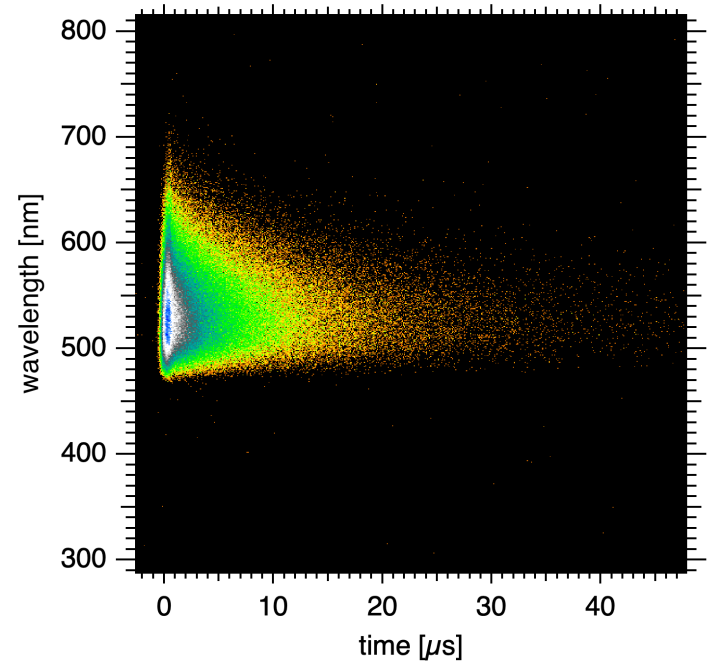

(b) $3 \mathrm{CzClIPN}$ in PS

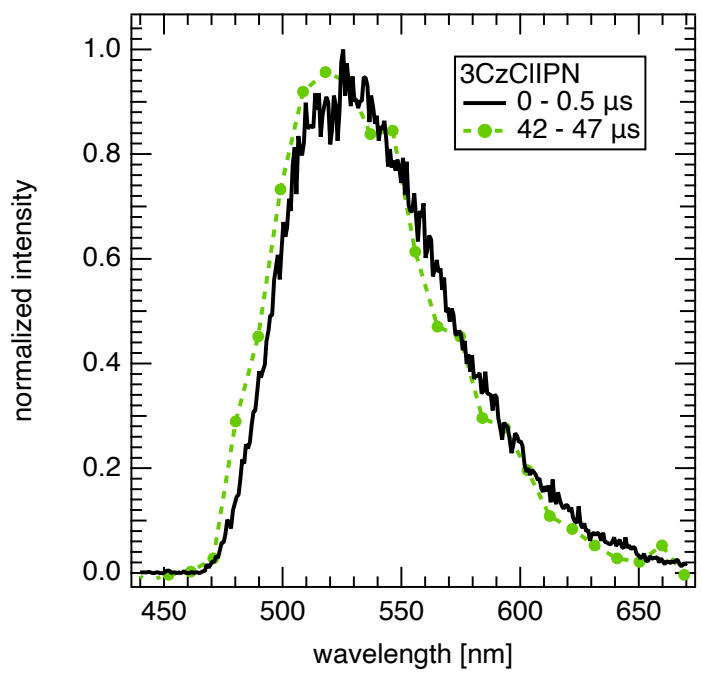

(d) $3 \mathrm{CzClIPN}$ in PS

FIG. 9: Time-resolved spectra

Streak measurements were performed on a Hamamatsu C10910-05 camera. Films were excited at $405 \mathrm{~nm}$ with a pulse rate of $10 \mathrm{kHz}$ (Light Conversion, Pharos laser with Orpheus OPA) in an evacuated cryostate (JANIS, ST-500). Delayed spectra were binned to 12 data points. Note, that the PL part of the decay is distorted when measuring the full decay in a $50 \mu$ s window, as the IRF is longer than the nanosecond decay. To evaluate transients, the PL must be additionally measured in a smaller time window (50-200 ns) at the same pulse rate. 


\section{QUANTUM CHEMICAL CALCULATIONS}

Structural models of $4 \mathrm{CzIPN}$ and $3 \mathrm{CzIPN}$ were generated with GaussView. ${ }^{2}$ Their ground state geometries were optimized with density functional theory (DFT) calculations utilizing the B3LYP functional, ${ }^{3-5}$ which has been applied in previous studies of TADF emitter materials. ${ }^{6-12}$ Furthermore, Grimme dispersion correction with Becke-Johnson damping (D3BJ) ${ }^{13,14}$ was employed to improve the description of the interaction between the carbazole units. ${ }^{15,16}$ During the optimizations, the double- $\zeta$ Pople style basis set $6-31+\mathrm{G}^{*}$ was utilized. ${ }^{17}$ Calculations of vibrational frequencies on the same level of theory were realized to check that the optimized structures correspond to minima on the potential energy surface. The subsequent time-dependent DFT (TD-DFT) calculations with and without TammDancoff approximation (TDA) for the first 20 excited states with singlet and triplet multiplicity were performed with the same basis set employing either the B3LYP functional as for the structure optimizations or the range-separated hybrid functional CAM-B3LYP. ${ }^{18}$ The described quantum chemical calculations were realized with the Gaussian 16 software package. ${ }^{19}$

Furthermore, the Orca program ${ }^{20,21}$ was used for the sTD-DFT and sTDA calculations ${ }^{22-24}$ with the same functionals and the somewhat larger basis set def2-SVPD. ${ }^{25}$ In these calculations, all excited states up to $10 \mathrm{eV}$ were determined. ${ }^{24,25}$ In addition, Turbomole ${ }^{26,27}$ was employed for the excited state calculations with the approximate coupled cluster singles and doubles $(\mathrm{CC} 2)^{28-30}$ as well as the algebraic diagrammatic construction to second order methods $(\mathrm{ADC}(2)) .{ }^{31,32}$ Besides the unscaled methods, also the spin-component scaled (SCS) variants ${ }^{33,34}$ were ap- $^{2}$ plied. These calculations utilized also the def2-SVPD basis set ${ }^{25}$ in combination with the corresponding auxiliary basis set for the resolution-of-identity (RI) approximation ${ }^{35}$ and an SCF convergence criterion of $10^{-8}$ Hartree. Furthermore, core orbitals were frozen and only the first three excited states of singlet and triplet multiplicity were determined.

Finally wavefunction analysis was performed with the TheoDORE software package. ${ }^{36,37}$ The obtained natural transition orbitals (NTOs) were visualized with Jmol. ${ }^{38}$

\section{FURTHER RESULTS FROM EXCITED STATE CALCULATIONS}

\begin{tabular}{lcclcll}
\multicolumn{1}{c}{4 CzIPN } & \multicolumn{5}{c}{$3 \mathrm{CzClIPN}$} \\
& $\begin{array}{c}\Delta E_{\mathrm{ST}} \\
\text { method }\end{array}$ & $S_{1}$ & $T_{1}$ & $\Delta E_{\mathrm{ST}}$ & $S_{1}$ & $T_{1}$ \\
{$[\mathrm{meV}]$} & {$[\mathrm{eV}]$} & {$[\mathrm{eV}]$} & {$[\mathrm{meV}]$} & {$[\mathrm{eV}]$} & {$[\mathrm{eV}]$} \\
\hline \hline B3LYP & & & & & & \\
TD-DFT & 146 & 2.558 & 2.412 & 103 & 2.460 & 2.356 \\
TDA & 154 & 2.578 & 2.423 & 108 & 2.473 & 2.365 \\
sTD-DFT & 46 & 2.411 & 2.365 & 41 & 2.341 & 2.300 \\
sTDA & 56 & 2.421 & 2.365 & 47 & 2.347 & 2.300 \\
\hline CAM-B3LYP & & & & & & \\
TD-DFT & 693 & 3.324 & 2.631 & 589 & 3.259 & 2.670 \\
TDA & 359 & 3.359 & 3.000 & 232 & 3.279 & 3.047 \\
sTD-DFT & 77 & 3.056 & 2.979 & 49 & 3.013 & 2.964 \\
sTDA & 87 & 3.067 & 2.980 & 54 & 3.019 & 2.965 \\
\hline Post-HF & & & & & & \\
ADC(2) & 45 & 2.765 & 2.719 & 20 & 2.770 & 2.751 \\
SCS-ADC(2) & 42 & 3.115 & 3.072 & 15 & 3.137 & 3.122 \\
CC2 & 43 & 2.842 & 2.798 & 22 & 2.847 & 2.824 \\
SCS-CC2 & 38 & 3.179 & 3.140 & 16 & 3.204 & 3.188
\end{tabular}

TABLE II: Vertical singlet-triplet gaps and energies of the first excited singlet and triplet states relative to the optimized ground state.

To further assess the sTD-DFT and sTDA as well as the (SCS-)CC2 results, we performed additional calculations via regular TD-DFT with and without TDA and via $\mathrm{ADC}(2)$ with and without spin-component scaling. As summarized in table II, the computed values for the vertical energy gap between $T_{1}$ and $S_{1}\left(\Delta E_{\mathrm{ST}}\right)$ strongly depend on the choice of the functional in TD-DFT. The global hybrid functional B3LYP with $20 \%$ exact exchange overestimates 
this splitting by a factor of approximately 2 relative to the experimental values, whereas the range-separated CAMB3LYP functional yields significantly larger values. Our results for $4 \mathrm{CzIPN}$ corroborate the findings that the choice of functional shows a pronounced effect on $\Delta E_{\mathrm{ST}}$ and that the results from the employed global hybrid functional are in better agreement with experiments than from the range-separated one, which have first been reported by Adachi et al. $^{39}$

Furthermore, the application of the TDA in the excited state calculations improves the results only in case of CAMB3LYP, but the computed values for the vertical $\Delta E_{\mathrm{ST}}$ are still much larger than with the B3LYP functional. For the latter, TDA does not lead to significant improvements. Relative to the results obtained with regular TD-DFT, the semiempirical sTD-DFT and sTDA approaches proposed by Grimme and coworkers ${ }^{22-24}$ yield values of $\Delta E_{\mathrm{ST}}$, which are in better agreement with the Post-Hartree-Fock methods. Also these values do not exhibit such a pronounced dependence on the chosen functional as for the regular TD-DFT/TDA calculations, but the individual energies of the $S_{1}$ and $T_{1}$ states behave more similar.

The origin of the good performance of the semiempirical sTD-DFT and sTDA methods for $\Delta E_{\mathrm{ST}}$ might be traced back to the fact that they are based on regular DFT ground state calculations, but the parameters employed in the excited state calculations were fitted to reproduce excitation energies obtained from SCS-CC2 calculations. ${ }^{24}$ To assess the performance and reliability of this and related Post-Hartree-Fock methods, we performed corresponding calculations for $4 \mathrm{CzIPN}$ and $3 \mathrm{CzClIPN}$. For this purpose, we employed the two approximate second order methods CC2 and ADC(2) with and without SCS. All four methods result in a similar value for the difference of $\Delta E_{\mathrm{ST}}$ between $4 \mathrm{CzIPN}$ and $3 \mathrm{CzClIPN}$ ranging from 21 to $27 \mathrm{meV}$. Furthermore, the individual values are below $50 \mathrm{meV}$. In contrast to this, the vertical excitation energies of $S_{1}$ and $T_{1}$ exhibit a larger variance of around $0.4 \mathrm{eV}$, which is reminiscent of the functional dependence in case of the DFT-based calculations. Overall, the employed second order methods result in a consistent picture for $\Delta E_{\mathrm{ST}}$.

\section{POPULATION ANALYSIS}

To better understand the increase in charge transfer character from $4 \mathrm{CzIPN}$ to $3 \mathrm{CzClIPN}$, the individual populations of the excited electron and hole for the three fragments can be analyzed for each state, see table III. The hole population on fragment $1\left(h_{1}\right)$ and the electron population on fragment $2\left(e_{2}\right)$ increase, whereas the hole population on fragment $2\left(h_{2}\right)$ and electron population on fragment $1\left(e_{1}\right)$ decrease. Therefore, the hole becomes more localized on fragment 1 and the electron on fragment 2. In contrast to this, the populations on fragment 3 remain nearly constant. Therefore, also this analysis shows that the localization of the excited electron and of the hole on different fragments is favored for $3 \mathrm{CzClIPN}$ relative to $4 \mathrm{CzIPN}$ resulting in a smaller overlap of the corresponding wavefunctions and a decrease of $\Delta E_{\mathrm{ST}}$.

\begin{tabular}{|l|l|l|l|l|}
\hline & $4 \mathrm{CzIPN}$ & & $3 \mathrm{CzClIPN}$ & \\
\hline & $T_{1}$ & $S_{1}$ & $T_{1}$ & $S_{1}$ \\
\hline$h_{1}$ & 0.739 & 0.798 & 0.822 & 0.848 \\
$e_{1}$ & 0.164 & 0.165 & 0.113 & 0.113 \\
$h_{2}$ & 0.207 & 0.146 & 0.123 & 0.103 \\
$e_{2}$ & 0.620 & 0.618 & 0.664 & 0.665 \\
$h_{3}$ & 0.008 & 0.008 & 0.010 & 0.004 \\
$e_{3}$ & 0.170 & 0.169 & 0.177 & 0.178 \\
\hline
\end{tabular}

TABLE III: Electron $(e)$ and hole $(h)$ populations of the first excited singlet states $\left(S_{1}\right)$ and the first triplet state $\left(T_{1}\right)$ divided into three fragments for the two investigated molecules obtained from the SCS-CC2 calculations. The first fragment consists of the carbazole units, the second one of the benzene ring and in case of $3 \mathrm{CzClIPN}$ also of the $\mathrm{Cl}$ atom, and the third one of the cyano groups. 
1 T. Kobayashi, A. Niwa, K. Takaki, S. Haseyama, T. Nagase, K. Goushi, C. Adachi, and H. Naito, Physical Review Applied 7, 034002 (2017).

${ }^{2}$ R. Dennington, T. Keith, and J. Millam, GaussView Version 5.0.9. Semichem Inc. Shawnee Mission KS (2009).

3 A. D. Becke, Physical Review A 38, 3098 (1988).

${ }^{4}$ C. Lee, W. Yang, and R. G. Parr, Physical Review B 37, 785 (1988).

5 A. D. Becke, The Journal of Chemical Physics 98, 5648 (1993).

${ }^{6}$ K. Liang, C. Zheng, K. Wang, W. Liu, Z. Guo, Y. Li, and X. Zhang, Physical Chemistry Chemical Physics 18, 26623 (2016).

7 P. De Silva, Journal of Physical Chemistry Letters 10, 5674 (2019).

8 C. Wang, K. Zhou, S. Huang, and Q. Zhang, Journal of Physical Chemistry C 123, 13869 (2019).

${ }^{9}$ W. Zhang, H. Song, J. Kong, Z. Kuang, M. Li, Q. Guo, C.-f. Chen, and A. Xia, The Journal of Physical Chemistry C 123, $19322(2019)$.

10 S. Montanaro, A. J. Gillett, S. Feldmann, E. W. Evans, F. Plasser, R. H. Friend, and I. A. Wright, Physical Chemistry Chemical Physics 21, 10580 (2019).

11 S. Weissenseel, N. A. Drigo, L. G. Kudriashova, M. Schmid, T. Morgenstern, K.-H. Lin, A. Prlj, C. Corminboeuf, A. Sperlich, W. Brütting, M. K. Nazeeruddin, and V. Dyakonov, The Journal of Physical Chemistry C 123, 27778 (2019).

12 S. Izumi, H. F. Higginbotham, A. Nyga, P. Stachelek, N. Tohnai, P. de Silva, P. Data, Y. Takeda, and S. Minakata, Journal of the American Chemical Society, jacs.9b11578 (2020).

13 S. Grimme, J. Antony, S. Ehrlich, and H. Krieg, J. Chem. Phys. 132, 154104 (2010).

14 S. Grimme, S. Ehrlich, and L. Goerigk, Journal of Computational Chemistry 32, 1456 (2011).

15 M. Moral, L. Muccioli, W. J. Son, Y. Olivier, and J. C. Sancho-Garcia, Journal of Chemical Theory and Computation 11, $168(2015)$.

16 Y. Noguchi and O. Sugino, Journal of Physical Chemistry C 121, 20687 (2017).

17 W. J. Hehre, R. Ditchfield, and J. A. Pople, J. Chem. Phys. 56, 2257 (1977).

18 T. Yanai, D. P. Tew, and N. C. Handy, Chemical Physics Letters 393, 51 (2004).

19 M. J. Frisch, G. W. Trucks, H. B. Schlegel, G. E. Scuseria, M. A. Robb, J. R. Cheeseman, G. Scalmani, V. Barone, G. A. Petersson, H. Nakatsuji, X. Li, M. Caricato, A. V. Marenich, J. Bloino, B. G. Janesko, R. Gomperts, B. Mennucci, H. P. Hratchian, J. V. Ortiz, A. F. Izmaylov, J. L. Sonnenberg, D. Williams-Young, F. Ding, F. Lipparini, F. Egidi, J. Goings, B. Peng, A. Petrone, T. Henderson, D. Ranasinghe, V. G. Zakrzewski, J. Gao, N. Rega, G. Zheng, W. Liang, M. Hada, M. Ehara, K. Toyota, R. Fukuda, J. Hasegawa, M. Ishida, T. Nakajima, Y. Honda, O. Kitao, H. Nakai, T. Vreven, K. Throssell, J. A. Montgomery, Jr., J. E. Peralta, F. Ogliaro, M. J. Bearpark, J. J. Heyd, E. N. Brothers, K. N. Kudin, V. N. Staroverov, T. A. Keith, R. Kobayashi, J. Normand, K. Raghavachari, A. P. Rendell, J. C. Burant, S. S. Iyengar, J. Tomasi, M. Cossi, J. M. Millam, M. Klene, C. Adamo, R. Cammi, J. W. Ochterski, R. L. Martin, K. Morokuma, O. Farkas, J. B. Foresman, and D. J. Fox, Gaussian 16, Revision C.01. Gaussian Inc. Wallingford CT (2019).

${ }^{20}$ F. Neese, WIREs Comput. Mol. Sci. 2, 73 (2012).

21 F. Neese, Wiley Interdisciplinary Reviews: Computational Molecular Science 8, e1327 (2018).

22 S. Grimme, The Journal of Chemical Physics 138, 244104 (2013).

23 C. Bannwarth and S. Grimme, Computational and Theoretical Chemistry 1040-1041, 45 (2014).

${ }^{24}$ T. Risthaus, A. Hansen, and S. Grimme, Phys. Chem. Chem. Phys. 16, 14408 (2014).

25 D. Rappoport and F. Furche, Journal of Chemical Physics 133, 134105 (2010).

26 F. Furche, R. Ahlrichs, C. Hättig, W. Klopper, M. Sierka, and F. Weigend, Wiley Interdisciplinary Reviews: Computational Molecular Science 4, 91 (2014).

27 TURBOMOLE V7.2 2017, a development of University of Karlsruhe and Forschungszentrum Karlsruhe GmbH, 1989-2007, TURBOMOLE GmbH, since 2007. Available from http://www.turbomole.com (Accessed: 23/01/2020).

28 O. Christiansen, H. Koch, P. Jørgensen, and T. Helgaker, Chemical Physics Letters 263, 530 (1996).

29 C. Hättig and F. Weigend, Journal of Chemical Physics 113, 5154 (2000).

30 C. Hättig and K. Hald, Physical Chemistry Chemical Physics 4, 2111 (2002).

31 A. B. Trofimov and J. Schirmer, Journal of Physics B: Atomic, Molecular and Optical Physics 28, 2299 (1995).

32 C. Hättig, Advances in Quantum Chemistry 50, 37 (2005).

33 S. Grimme, Journal of Chemical Physics 118, 9095 (2003).

34 A. Hellweg, S. A. Grün, and C. Hättig, Physical Chemistry Chemical Physics 10, 4119 (2008).

35 A. Hellweg and D. Rappoport, Physical Chemistry Chemical Physics 17, 1010 (2015).

${ }^{36}$ F. Plasser and H. Lischka, Journal of Chemical Theory and Computation 8, 2777 (2012).

37 F. Plasser, TheoDORE 1.6: a package for theoretical density, orbital relaxation, and exciton analysis. Available from http://theodore-qc.sourceforge.net (Accessed: 23/01/2020).

38 Jmol: an open-source Java viewer for chemical structures in 3D, http://www.jmol.org/ (Accessed: 23/01/2020).

39 H. Uoyama, K. Goushi, K. Shizu, H. Nomura, and C. Adachi, Nature 492, 234 (2012) 